\title{
Efficient Estimation of the Semiparametric Spatial Autoregressive Model
}

\author{
P.M. Robinson* \\ Department of Economics, London School of Economics, \\ Houghton Street, London WC2A 2AE, UK
}

Discussion paper

No. EM/2007/515

February 2007
The Suntory Centre

Suntory and Toyota International Centres for Economics and Related Disciplines London School of Economics and Political Science Houghton Street London WC2A $2 \mathrm{AE}$ Tel: 02079556679

\footnotetext{
* $\vee$ Corresponding author: Tel. +44-20-7955-7516; fax: +44-20-7955-6592. E-mail address: p.m.robinson@lse.ac.uk.
} 


\begin{abstract}
Efficient semiparametric and parametric estimates are developed for a spatial autoregressive model, containing nonstochastic explanatory variables and innovations suspected to be non-normal. The main stress is on the case of distribution of unknown, nonparametric, form, where series nonparametric estimates of the score function are employed in adaptive estimates of parameters of interest. These estimates are as efficient as ones based on a correct form, in particular they are more efficient than pseudo-Gaussian maximum likelihood estimates at non-Gaussian distributions. Two different adaptive estimates are considered. One entails a stringent condition on the spatial weight matrix, and is suitable only when observations have substantially many "neighbours". The other adaptive estimate relaxes this requirement, at the expense of alternative conditions and possible computational expense. A Monte Carlo study of finite sample performance is included.
\end{abstract}

JEL Classifications: C13; C14; C21

Keywords: Spatial autoregression; Efficient estimation; Adaptive estimation; Simultaneity bias.

(c) The author. All rights reserved. Short sections of text, not to exceed two paragraphs, may be quoted without explicit permission provided that full credit, including (๑) notice, is given to the source. 


\section{Introduction}

Spatial autoregressive models have proved a popular basis for statistical inference on spatial econometric data. Much of the spatial statistics literature has focussed on data recorded on a lattice, that is, it is regularly-spaced in two or more dimensions. This is an uncommon framework in economics, at best an approximation. Data recorded over geographical space are apt to be very irregularly spaced, for example when observations are across cities or towns, or aggregated on possibly contiguous regions, such as provinces or countries. A recent review of spatial econometrics is Arbia (2006). A statistical model that adequately describes dependence as a function of geographic distance is apt to be complicated, especially in the second kind of situation, and derivation of rules of large sample statistical inference under plausible conditions difficult; even for time series data, where there is a single dimension, inference in irregularlyspaced settings is not very well developed. On the other hand, cross-sectional correlation has been measured as a function of "economic distance", not necessarily in a geographic setting. Spatial autoregressive models are applicable in all these circumstances.

We wish to model an $n \times 1$ vector of observations $y=\left(y_{1}, \ldots, y_{n}\right)^{T}$, on a scalar variate $y_{i}, T$ indicating transposition. We have an $n \times k$ matrix of constants $X=\left(x_{1}, \ldots, x_{n}\right)^{T}, x_{i}$ being a $k \times 1$ vector, where $k \geq 1$. Let $\varepsilon=\left(\varepsilon_{1}, \ldots, \varepsilon_{n}\right)^{T}$ be an $n \times 1$ vector of unobservable random variables, that are independent and identically distributed (iid) with zero mean and unit variance. Let $l_{n}$ be the $n \times 1$ vector $(1, \ldots, 1)^{T}$. Finally, let $W$ be a given $n \times n$ "weight" matrix, having zero diagonal elements and being row-normalized such that elements of each row sum to 1 , so

$$
W l_{n}=l_{n} .
$$

We assume that, for some scalars $\mu_{0}, \sigma_{0}$ and $\lambda_{0}$, and some $k \times 1$ vector $\beta_{0}$,

$$
y=\mu_{0} l_{n}+\lambda_{0} W y+X \beta_{0}+\sigma_{0} \varepsilon .
$$

Here, $\mu_{0}$ and $\sigma_{0}>0$ are unknown nuisance parameters, representing intercept and scale respectively: they can be estimated, but our focus is on estimating $\theta_{0}=\left(\lambda_{0}, \beta_{0}^{T}\right)^{T}$, where $\lambda_{0} \in(-1,1)$ and $\beta_{0}$ is non-null. It is taken for granted that no restrictions link $\theta_{0}, \mu_{0}$ and $\sigma_{0}$. The matrix $\left(l_{n}, X\right)$ is assumed to have full column rank for sufficiently large $n, k \geq 1$ implying at least one non-intercept regressor.

The practitioner must choose $W$. In view of (1.1), we can define it in terms of an underlying non-negative inverse "distance" measure $d_{i j}$ such that $W$ has $(i, j)$-th element

$$
w_{i j}=\frac{d_{i j}}{\sum_{h=1}^{n} d_{i h}} .
$$

However, the "distance" terminology is not taken to imply that $W$ is necessarily a symmetric matrix. Though we have mostly suppressed reference to the data 
size $n$ for concise notation, the row-normalization of $W$ implies that as $n \rightarrow \infty$, $y$ must be treated like a triangular array.

In recent years considerable progress has been made in the econometric literature on developing asymptotic properties of various estimates for (1.2). The ordinary least squares (OLS) estimate of $\theta_{0}$ in (1.2) (with $\mu_{0}$ unknown) is generally inconsistent, because, for each $i$, the $i$-th element of $W y$ is correlated with $\varepsilon_{i}$, contrasting with the corresponding classical dynamic time series model. In case of multilateral autoregressive models on a lattice, Whittle (1954) corrected the problem by using Gaussian maximum likelihood (ML) estimation. Correspondingly, Lee (2004) has established $n^{\frac{1}{2}}$-consistency and asymptotic normality and efficiency of Gaussian ML in (1.2). An alternative, if generally sub-optimal solution, is instrumental variables, justified by Kelejian and Prucha $(1998,1999)$, Lee (2003), Kelejian, Prucha and Yuzefovich (2003). On the other hand, returning to OLS, Lee (2002) noticed that this can still be consistent, and even $n^{\frac{1}{2}}$-consistent and asymptotically normal and efficient, under suitable conditions on $W$. In particular, he showed that consistency is possible if the $d_{i j}$ in (1.3) are uniformly bounded and the $\sum_{j=1}^{n} d_{i j}$ tend to infinity with $n$, and $n^{\frac{1}{2}}$-consistent if the latter sums tend to infinity faster than $n^{\frac{1}{2}}$.

This can be simply illustrated in terms of a $W$ employed in an empirical example of Case (1992), and stressed by Lee (2002). Data are recorded across $p$ districts, in each of which are $q$ farmers. Independence between farmers in different districts is assumed, and neighbours at each farm within a district are given equal weight. Due to (1.1) we have

$$
W=I_{p} \otimes(q-1)^{-1}\left(l_{q} l_{q}^{T}-I_{q}\right) .
$$

In this setting, OLS is consistent if

$$
q \rightarrow \infty, \quad \text { as } n \rightarrow \infty,
$$

and $n^{\frac{1}{2}}$-consistent if

$$
q / p \rightarrow \infty \text { as } n \rightarrow \infty
$$

Lee (2004), on the other hand, interpreted his procedure not just as ML under Gaussianity, but also pseudo-ML under departures from Gaussianity, as has been done in many other settings. However, though $n^{\frac{1}{2}}$-consistency and asymptotic normality is still relevant, asymptotic efficiency is not. When datasets are not very large, precision is important, and since there is often reason not to take Gaussianity seriously, it is desirable to develop estimates which are efficient in non-Gaussian populations.

As typically in time series models, building a non-Gaussian likelihood is most easily approached by introducing a non-normal distribution for the iid $\varepsilon_{i}$ in (1.2) (for example a Student- $t$ distribution). Such a distribution may also involve unknown nuisance parameters, to be estimated alongside the original ones. We present limit distributional results for one-step Newton approximations to ML estimates in this case. However, there is rarely a strong reason for picking a particular parametric form for the underlying innovation density, and if this is 
mis-specified not only would the estimates not be asymptotically efficient (or necessarily more efficient than the Gaussian pseudo-ML estimates of Lee (2004)), but in some cases they may actually be inconsistent. As in other statistical problems, these drawbacks, as well as possible computational complications, do much to explain the popularity of Gaussian pseudo-ML procedures, and approximations to them.

On the other hand, the ability to "adapt" to distribution of unknown form is well-established in a number of other statistical and econometric models. Here the density $f$ of $\varepsilon_{i}$ in (1.2) is nonparametric, so (1.2) is a semiparametric model; $f$ is estimated by smoothing, and a suitable implementation provides parameter estimates that are $n^{\frac{1}{2}}$-consistent and normal, and asymptotically as efficient as ones based on a correctly parameterized $f$. This was demonstrated by Stone (1975), for the simple location model with iid data, and then by Bickel (1982), Newey (1988) for regression models with iid errors, and by other authors in various other models. ("Partially" adaptive estimates have been considered by Potscher and Pruscha (1986), for example.) The main focus of the present paper is to develop efficient estimates of the vector $\theta_{0}$ in (1.2). The ability to adapt in (1.2) is not guaranteed. Our first result requires similar conditions on $W$ to those Lee (2002) imposed in showing $n^{\frac{1}{2}}$-consistency of OLS (i.e. (1.6) in case (1.4)). Our second result employs a bias-reduced estimate that, in case (1.4), requires only (1.5), though either $W$ has also to be symmetric (as in (1.4)) or $\varepsilon_{i}$ has to be symmetrically distributed.

Our efficient estimates of $\theta_{0}$ are described in the following section. Regularity conditions and theorem statements are presented in Section 3. Section 4 consists of a Monte Carlo study of finite sample behaviour. Proofs are left to appendices.

\section{Efficient Estimates}

It is possible to write down an objective function that is a form of likelihood, employing a smoothed nonparametric estimate of the density $f$ of the $\varepsilon_{i}$. However, not only is this liable to be computationally challenging to optimize, but derivation of asymptotic properties would be a lengthy business since, as is common with implicitly-defined extremum estimation, proof of $n^{\frac{1}{2}}$-consistency and asymptotic normality has to be preceded by a consistency proof. The latter can be by-passed by the familiar routine of taking one Newton-type iterative step, based on the aforementioned "likelihood", from an initial $n^{\frac{1}{2}}$-consistent estimate. This strategy is followed virtually uniformly in the adaptive estimation literature.

It leads to the need to nonparametrically estimate not $f(s)$, but the score function

$$
\psi(s)=-\frac{f^{\prime}(s)}{f(s)},
$$

where throughout the paper the prime denotes differentiation. The bulk of work on adaptive estimation uses kernel estimates of $f$ and $f^{\prime}$. Kernel estimation is 
very familiar in econometrics, and can have important advantages. However, separate estimation of $f$ and $f^{\prime}$ is necessary, and the resulting estimate of $\psi$ is somewhat cumbersome.

More seriously, since $f$ is liable to become small, use of an estimate in the denominator of (2.1) is liable to cause instability. It also causes technical difficulty, and typically some form of trimming is introduced. This requires introduction of a user-chosen trimming number, itself a disincentive to the practitioner. In addition, kernel-based adaptive estimates have, for technical reasons, featured sample-splitting (use of one part of the sample in the nonparametric estimation, and the other for the final parametric estimation) which is wasteful of data and introduces a further ambiguity, as well as the artificial device of discretizing the initial parameter estimate.

These drawbacks are overcome by series estimation of $\psi$, as proposed by Beran (1976) in a time series autoregressive setting. Let $\phi_{\ell}(s), \ell=1,2, \ldots$, be a sequence of smooth functions. For some user-chosen integer $L \geq 1$, where $L=L_{n}$ will be regarded as increasing slowly with $n$, define the vectors

$$
\begin{aligned}
\phi^{(L)}(s) & =\left(\phi_{1}(s), \ldots, \phi_{L}(s)\right)^{T}, \bar{\phi}^{(L)}(s)=\phi^{(L)}(s)-E\left\{\phi^{(L)}\left(\varepsilon_{i}\right)\right\}, \\
\phi^{\prime(L)}(s) & =\left(\phi_{1}^{\prime}(s), \ldots, \phi_{L}^{\prime}(s)\right)^{T} .
\end{aligned}
$$

Consider for $\psi(s)$ first the parametric form

$$
\psi(s)=\bar{\phi}^{(L)}(s)^{T} a^{(L)},
$$

where $a^{(L)}=\left(a_{1}, \ldots, a_{L}\right)^{T}$ is a vector with unknown elements. The meancorrection in (2.2) imposes the restriction $E\left\{\psi\left(\varepsilon_{i}\right)\right\}=0$. Under mild conditions on $f$, integration-by-parts allows $a^{(L)}$ to be identified by

$$
a^{(L)}=\left[E\left\{\bar{\phi}^{(L)}\left(\varepsilon_{i}\right) \bar{\phi}^{(L)}\left(\varepsilon_{i}\right)^{T}\right\}\right]^{-1} E\left\{\phi^{\prime(L)}\left(\varepsilon_{i}\right)\right\} .
$$

Given a vector of observable proxies $\tilde{\varepsilon}=\left(\tilde{\varepsilon}_{1}, \ldots, \tilde{\varepsilon}_{n}\right)^{T}$, we approximate $a^{(L)}$ by $\tilde{a}^{(L)}(\tilde{\varepsilon})$, where, for a generic vector $q=\left(q_{1}, \ldots, q_{n}\right)^{T}$,

$$
\tilde{a}^{(L)}(q)=W^{(L)}(q)^{-1} w^{(L)}(q),
$$

with

$$
\begin{aligned}
W^{(L)}(q) & =\frac{1}{n} \sum_{i=1}^{n} \Phi^{(L)}\left(q_{i}\right) \Phi^{(L)}\left(q_{i}\right)^{T} \\
w^{(L)}(q) & =\frac{1}{n} \sum_{i=1}^{n} \phi^{\prime(L)}\left(q_{i}\right)
\end{aligned}
$$

and

$$
\Phi^{(L)}\left(q_{i}\right)=\phi^{(L)}\left(q_{i}\right)-\frac{1}{n} \sum_{j=1}^{n} \phi^{(L)}\left(q_{j}\right) .
$$


Then defining

$$
\psi^{(L)}\left(q_{i} ; \tilde{a}^{(L)}(q)\right)=\Phi^{(L)}\left(q_{i}\right)^{T} \tilde{a}^{(L)}(q),
$$

$\tilde{\psi}_{i L}=\psi^{(L)}\left(\tilde{\varepsilon}_{i} ; \tilde{a}^{(L)}(\tilde{\varepsilon})\right)$ is a proxy for $\psi\left(\varepsilon_{i}\right)$, and is inserted in the Newton step for estimating the unknown parameters.

However, Beran's (1976) asymptotic theory assumed that, for the chosen $L$, (2.3) correctly specifies $\psi(s)$. This amounts almost to a parametric assumption on $f$ : indeed, for $L=1$ and $\phi_{1}(s)=s,(2.3)$ is just the score function for the standard normal distribution. Newey (1988) considerably developed the theory by allowing $L$ to go to infinity slowly with $n$, so that the right hand side of (2.3) approximates (an infinite series representation of) $\psi(s)$. In regression with independent cross-sectional observations he established analogous adaptivity results to those of Bickel (1982), using kernel estimation of $\psi$. Robinson (2005) developed Newey's (1988) asymptotic theory further, in the context of stationary and nonstationary fractional time series models. In the asymptotic theory, $L$ can be regarded as a smoothing number analogous to that used in a kernel approach, but no other user-chosen numbers or arbitrary constructions are required in the series approach, where indeed some regularity conditions are a little weaker than those in the kernel-based literature.

We follow the series estimation approach here, and for ease of reference mainly follow the notation of Robinson (2005). Consider the $n \times 1$ vector

$$
e(\theta)=\left(e_{1}(\theta), \ldots, e_{n}(\theta)\right)^{T}=(I-\lambda W) y-X \beta,
$$

for $\theta=\left(\lambda, \beta^{T}\right)^{T}$, and any scalar $\lambda$ and $k \times 1$ vector $\beta$. From (1.2)

$$
\sigma_{0} \varepsilon=e\left(\theta_{0}\right)-E\left\{e\left(\theta_{0}\right)\right\} .
$$

Accordingly, given an initial estimate $\tilde{\theta}$ of $\theta_{0}$, consider as a proxy for the vector $\sigma_{0} \varepsilon$ the vector $E(\tilde{\theta})$, where

$$
E(\theta)=e(\theta)-l_{n} \frac{1}{n} \sum_{i=1}^{n} e_{i}(\theta) .
$$

We can estimate $\sigma_{0}^{2}$ by $\tilde{\sigma}^{2}=\tilde{\sigma}^{2}(\tilde{\theta})$, where

$$
\tilde{\sigma}^{2}(\theta)=\frac{1}{n} E(\theta)^{T} E(\theta)
$$

Thus our proxy $\tilde{\varepsilon}$ for $\varepsilon$ is given by

$$
\tilde{\varepsilon}=E(\tilde{\theta}) / \tilde{\sigma} .
$$

We find it convenient to write

$$
\tilde{\psi}_{i L}=\tilde{\psi}_{i L}(\tilde{\theta}, \tilde{\sigma}),
$$

where

$$
\tilde{\psi}_{i L}(\theta, \sigma)=\Phi^{(L)}\left(E_{i}(\theta) / \sigma\right)^{T} \tilde{a}^{(L)}(E(\theta) / \sigma) .
$$


Now introduce the $(k+1) \times n$ matrix of derivatives

$$
e^{\prime}=\left[\frac{\partial e(\theta)}{\partial \lambda}, \frac{\partial e(\theta)}{\partial \beta^{T}}\right]^{T}
$$

in which

$$
\begin{aligned}
& \frac{\partial e(\theta)}{\partial \lambda}=-W y, \\
& \frac{\partial e(\theta)}{\partial \beta}=-X^{T},
\end{aligned}
$$

for all $\theta$. With $e_{i}^{\prime}$ denoting the $i$-th column of $e^{\prime}$ write

$$
E_{i}^{\prime}=e_{i}^{\prime}-\frac{1}{n} \sum_{j=1}^{n} e_{j}^{\prime}
$$

Now define

$$
R=\sum_{i=1}^{n} E_{i}^{\prime} E_{i}^{\prime T}
$$

and

$$
r_{L}(\theta, \sigma)=\sum_{i=1}^{n} \tilde{\psi}_{i L}(\theta, \sigma) E_{i}^{\prime}
$$

and let

$$
\tilde{\mathcal{I}}_{L}(\theta, \sigma)=\frac{1}{n} \sum_{i=1}^{n} \tilde{\psi}_{i L}^{2}(\theta, \sigma)
$$

so $\mathcal{I}_{L}(\tilde{\theta}, \tilde{\sigma})$ estimates the information measure

$$
\mathcal{I}=E \psi\left(\varepsilon_{i}\right)^{2}
$$

Our first adaptive estimate of $\theta_{0}$ is

$$
\hat{\theta}_{A}=\tilde{\theta}-\left[\tilde{\mathcal{I}}_{L}(\tilde{\theta}, \tilde{\sigma}) R\right]^{-1} r_{L}(\tilde{\theta}, \tilde{\sigma})
$$

(There is a typographical error in the corresponding formula (2.2) of Robinson (2005): " + should be " - ".) Define

$$
s_{L}(\theta, \sigma)=r_{L}(\theta, \sigma)-\left[\begin{array}{c}
\operatorname{tr}\left\{W\left(I_{n}-\lambda W\right)^{-1}\right\} \\
0
\end{array}\right],
$$

so $s_{L}$ and $r_{L}$ differ only in their first element. Our second adaptive estimate of $\theta_{0}$ is

$$
\hat{\theta}_{B}=\hat{\theta}-\left[\tilde{\mathcal{I}}_{L}(\tilde{\theta}, \tilde{\sigma}) R\right]^{-1} s_{L}(\tilde{\theta}, \tilde{\sigma})
$$

Some practical issues are outstanding. One is choice of the functions $\phi_{\ell}(s)$. As in Newey (1988), Robinson (2005), we restrict to "polynomial" forms

$$
\phi_{\ell}(s)=\phi(s)^{\ell},
$$


for some chosen function $\phi(s)$. For example,

$$
\begin{aligned}
& \phi(s)=s, \\
& \phi(s)=\frac{s}{\left(1+s^{2}\right)^{\frac{1}{2}}},
\end{aligned}
$$

where the boundedness in (2.30) can help to reduce other technical assumptions. Next, the choice of $L$ is discussed by Robinson (2005); asymptotic theory provides little guidance, delivering an upper bound on the rate at which $L$ can increase with $h$, but no lower bound. Since the upper bound is only logarithmic in $h$, it seems that $L$ should be far smaller than $n$. Discussion of $\tilde{\theta}$ is postponed to the following section.

For completeness, we also consider the fully parametric case, where $f\left(s ; \tau_{0}\right)$ is a prescribed parametric form for $f(s)$, with $\tau_{0}$ an unknown $m \times 1$ vector, on the basis of which define $\widehat{\tau}=\underset{\mathcal{T}}{\arg \min } \sum_{i} \log f\left(E_{i}(\widetilde{\theta}) / \widetilde{\sigma} ; \tau\right)$ for a subset $\mathcal{T}$ of $\mathbb{R}^{m}$, and, with $\psi(s ; \tau)=\{(\partial / \partial s) f(s ; \tau)\} / f(s ; \tau)$,

$$
\begin{aligned}
\mathcal{I}(\theta, \sigma, \tau) & =n^{-1} \sum_{i} \psi\left(E_{i}(\theta) / \sigma ; \tau\right)^{2} \\
r(\theta, \sigma, \tau) & =\sum_{i} \psi\left(E_{i}(\theta) / \sigma ; \tau\right) E_{i}^{\prime}(\theta) .
\end{aligned}
$$

Define also

$$
s(\theta, \sigma, \tau)=r(\theta, \sigma, \tau)-\left[\begin{array}{c}
\operatorname{tr}\left\{W\left(I_{n}-\lambda W\right)^{-1}\right\} \\
0
\end{array}\right] .
$$

Our two parametric estimates are

$$
\begin{aligned}
& \hat{\theta}_{C}=\tilde{\theta}-[\tilde{\mathcal{I}}(\tilde{\theta}, \tilde{\sigma}, \widehat{\tau}) R]^{-1} r(\tilde{\theta}, \tilde{\sigma}, \widehat{\tau}), \\
& \hat{\theta}_{D}=\tilde{\theta}-[\tilde{\mathcal{I}}(\tilde{\theta}, \tilde{\sigma}, \widehat{\tau}) R]^{-1} s(\tilde{\theta}, \tilde{\sigma}, \widehat{\tau}),
\end{aligned}
$$

the second being a "bias-corrected" version of the first.

\section{Asymptotic Normality and Efficiency}

We introduce first the following regularity conditions.

Assumption 1: For all sufficiently large n, the weight matrix $W$ has nonnegative elements that are uniformly of order $O(1 / h)$, where $h=h_{n}$ increases with $n$ such that

$$
h / n^{\frac{1}{2}} \rightarrow \infty, \text { as } n \rightarrow \infty,
$$

and has zero diagonal, satisfies (1.1), and is such that the elements of $l_{n}^{T} W$ and $l_{n}^{T} S^{-1}$ are uniformly bounded, where $S=I_{n}-\lambda_{0} W$. 
Assumption 2: The elements of the $x_{i}$ are uniformly bounded constants, and the matrix

$$
\Omega=\lim _{n \rightarrow \infty} \frac{1}{n}\left[\begin{array}{c}
\left(G X \beta_{0}\right)^{T} \\
X^{T}
\end{array}\right]\left[G X \beta_{0}, X\right]
$$

exists and is positive definite, where $G=W S^{-1}$.

Assumption 3: The $\varepsilon_{i}$ are iid with zero mean and unit variance, and probability density function $f(s)$ that is differentiable, and

$$
0<\mathcal{I}<\infty .
$$

Assumption 4: $\phi_{\ell}(s)$ satisfies (2.28), where $\phi(s)$ is strictly increasing and thrice differentiable and is such that, for some $\kappa \geq 0, K<\infty$,

$$
\begin{aligned}
|\phi(s)| & \leq 1+|s|^{\kappa} \\
\left|\phi^{\prime}(s)\right|+\left|\phi^{\prime \prime}(s)\right|+\left|\phi^{\prime \prime \prime}(s)\right| & \leq C\left(1+|\phi(s)|^{K}\right),
\end{aligned}
$$

where $C$ is throughout a generic positive constant.

Denote by $\eta=1+2^{\frac{1}{2}} \bumpeq 2.414$ and $\varphi=\left(1+\left|\phi\left(s_{1}\right)\right|\right) /\left\{\phi\left(s_{2}\right)-\phi\left(s_{1}\right)\right\},\left[s_{1}, s_{2}\right]$ being an interval on which $f(s)$ is bounded away from zero.

\section{Assumption 5:}

$$
L \rightarrow \infty, \text { as } n \rightarrow \infty
$$

and either

(i) $\kappa=0, E \varepsilon_{i}^{4}<\infty$, and

$$
\liminf _{n \rightarrow \infty}\left(\frac{\log h}{L}\right)>4\{\log \eta+\max (\log \varphi, 0)\} \simeq 3.52+4 \max (\log \varphi, 0),
$$

or (ii) $\kappa>0$ for some $\omega>0$ the moment generating function $E\left(e^{t\left|\varepsilon_{i}\right|^{\omega}}\right)$ exists for some $t>0$, and

$$
\liminf _{n \rightarrow \infty}\left(\frac{\log h}{L \log L}\right) \geq \frac{4 \kappa(\omega+1)}{\omega},
$$

or (iii) $\kappa>0, \varepsilon_{i}$ is almost surely bounded, and

$$
\liminf _{n \rightarrow \infty}\left(\frac{\log h}{L \log L}\right) \geq 4 \kappa .
$$

Assumption 6: As $n \rightarrow \infty$

$$
\tilde{\theta}-\theta_{0}=O_{p}\left(n^{-\frac{1}{2}}\right), \quad \tilde{\sigma}^{2}-\sigma_{0}^{2}=O_{p}\left(n^{-\frac{1}{2}}\right) .
$$

The proof of the following theorem is left to Appendix A. 
Theorem A Let (1.2) hold with $\lambda_{0} \in(-1,1)$, and let Assumptions 1-6 hold. Then as $n \rightarrow \infty$

$$
n^{\frac{1}{2}}\left(\hat{\theta}_{A}-\theta_{0}\right) \rightarrow_{d} N\left(0, \frac{\sigma_{0}^{2}}{\mathcal{I}} \Omega^{-1}\right),
$$

where the limit variance matrix is consistently estimated by $\left(\tilde{\sigma}^{2} / \tilde{\mathcal{I}}_{L}(\tilde{\theta}, \tilde{\sigma})\right) n R^{-1}$.

Remark 1 For the Gaussian pseudo-ML estimate, Lee (2004) finds the limit variance matrix to be $\sigma^{2} \Omega^{-1}$. Since $\mathcal{I} \geq 1, \hat{\theta}_{A}$ achieves an efficiency improvement over this when $\varepsilon_{i}$ is non-Gaussian.

Remark 2 Various initial estimates satisfying Assumption 6 are available in the literature. This is the case under (3.1) if $\tilde{\theta}$ is OLS (see Lee, 2002). Other possibilities are the Gaussian pseudo-ML estimate, and various IV estimates.

Remark 3 In view of Assumption 2, $\beta_{0}$ cannot be null, so Theorem A cannot estimate $\lambda_{0}$ in the "pure" spatial autoregressive model without explanatory variables (cf. Lee (2004)). (Likewise we cannot extend (1.2) to allow $\varepsilon$ to be generated by a spatial autoregression with unknown autoregressive coefficient.) Thus Theorem A does not provide a test of $\beta_{0}=0$, though it can be used to test exclusion of a proper subset of the elements of $x_{i}$. It can also be used to test the hypothesis of no spatial dependence, $\lambda_{0}=0$, and in this case the limit distribution in the Theorem is true even if (3.1) does not hold, indeed $h$ can be regarded as fixed with respect to $n$, and so designs with only very few "neighbours" are covered. For non-Gaussian data, the tests provided by the Theorem are in general expected to be locally more powerful than ones based on existing estimates. Divergence of $h$ is needed for information matrix blockdiagonality (Lee, 2004), and the no-multicollinearity Assumption 2 is necessary for our results.

Remark 4 Assumption 1 is discussed by Lee (2002). In case $W$ is given by (1.4), $h_{n} \sim q$, so condition (3.1) is equivalent to (1.6), and the rest of Assumption 1 is satisfied.

Remark 5 Assumptions 3 and 4 are taken from Robinson (2005), where they are discussed. Assumption 5 is a modification of Assumption A9 of Robinson (2005). It differs in part with respect to $h$ replacing $n^{\frac{1}{2}}$ there and in this sense is slightly weaker than the latter assumption under our Assumption 6 . Note that the proofs in Robinson (2005) are harder with respect to the (long range) dependence there, while ours are harder with respect to the simultaneity, and these aspects influence the differences between the conditions in the two articles. The main implication of Assumption 5 is that if we choose bounded $\phi$ then a fourth moment condition on $\varepsilon_{i}$ suffices, with a relatively mild upper bound restriction on the rate of increase of $L$ (see (i)). For unbounded $\phi$, we have a choice between moment generating function (ii) and boundedness (iii) requirements on $\varepsilon_{i}$, where the condition on $L$ is weaker in the latter case, but still stronger than that of (i) of Assumption 5. 
Remark 6 It would be possible to obtain analogous results for a non-linear regression extension of (1.2), in which the elements of $X \beta_{0}$ are replaced by the nonlinear-in- $\beta_{0}$ functions $g\left(x_{i} ; \beta_{0}\right), i=1, \ldots, n$, where $g$ is a smooth function of known form. With respect to the initial estimate $\tilde{\theta}$ it would seem that non-linear least squares can be shown to satisfy Assumption 6 under (3.1), by extending the arguments of Lee (2002).

Remark 7 In practice further iteration of (2.25) may be desirable. This would not change the limit distribution, but can improve higher-order efficiency (Robinson, 1988).

By far the most potentially restrictive of the conditions underlying Theorem A is (3.1) of Assumption 1. It is never really possible to assess relevance of an asymptotic condition such as this to a given, finite, data set. However, if, in the simple case where $W$ is given by (1.4), $q$ is small relative to $p$, one expects that $\hat{\theta}_{A}$ may be seriously biased, and the normal approximation poor; the same will be true of OLS.

Results of Lee (2004) on the Gaussian pseudo-MLE hint at how it may be possible to relax (3.1). To best see this we temporarily modify the model (1.2) to

$$
y=\lambda_{0} W y+Z \gamma_{0}+\sigma_{0} \varepsilon .
$$

If an intercept is allowed, as in (1.2), then $l_{n}$ is a column of $Z, Z=\left(l_{n}, X\right)$, and $\gamma_{0}=\left(\mu_{0}, \beta_{0}^{T}\right)^{T}$. But it is also possible that no intercept is allowed, unlike in (1.2), in which case $Z=X$ and $\gamma_{0}=\beta_{0}$ (and $\mu_{0}=0$ ). The form (3.12) is the most usual in the literature. Lee (2004) shows the Gaussian pseudo-MLE $\hat{\xi}^{*}=\left(\hat{\lambda}^{*}, \hat{\gamma}^{* T}, \hat{\sigma}^{* 2}\right)^{T}$ of $\xi_{0}=\left(\lambda_{0}, \gamma_{0}^{T}, \sigma_{0}^{2}\right)^{T}$ is $n^{\frac{1}{2}}$-consistent and asymptotically normal, under mild conditions that do not even require that $h_{n}$ diverge (i.e. in (1.4), $q$ can remain fixed). However, even under Gaussianity, $\hat{\lambda}^{*}$ and $\hat{\sigma}^{* 2}$ are not independent in the limit distribution if $h_{n}$ does not diverge, suggesting that adaptive estimation of $\lambda_{0}, \gamma_{0}$ is not possible in this scenario. Lee (2004) finds, however, that the limit covariance matrix of $\hat{\xi}^{*}$ simplifies when

$$
h \rightarrow \infty, \text { as } n \rightarrow \infty,
$$

(i.e. (1.5) under (1.4)). His formulae indicate that $\left(\hat{\lambda}^{*}, \hat{\gamma}^{* T}\right)^{T}$ and $\hat{\sigma}^{* 2}$ will then be asymptotically independent if $E\left(\varepsilon_{i}^{3}\right) l_{n}^{T} G Z \gamma_{0} / n \rightarrow 0, E\left(\varepsilon_{i}^{3}\right) l_{n}^{T} / n \rightarrow 0$, as $n \rightarrow \infty$. This is true if $\varepsilon_{i}$ is normally distributed, and somewhat more generally, e.g. if $\varepsilon_{i}$ is symmetrically distributed. Reverting now to our model (1.2) and with $\left(\hat{\lambda}^{*}, \hat{\beta}^{* T}, \hat{\sigma}^{* 2}\right)$ denoting the Gaussian pseudo-MLE of $\left(\lambda_{0}, \beta_{0}^{T}, \sigma_{0}^{2}\right)$, analogously $\left(\hat{\lambda}^{*}, \hat{\beta}^{* T}\right)$ and $\hat{\sigma}^{* 2}$ are asymptotically independent if

$$
E\left(\varepsilon_{i}^{3}\right) l_{n}^{T} G H X \beta_{0} / n \rightarrow 0, \quad E\left(\varepsilon_{i}^{3}\right) l_{n}^{T} H X / n \rightarrow 0, \text { as } n \rightarrow \infty,
$$

where $H=I_{n}-l_{n} l_{n}^{T} / n$. The latter limit always holds (since $l_{n}^{T} H=0$ ), indeed the left-hand side is null for all $n$. The first limit holds if $W$ is symmetric 
(because (1.1) then implies $l_{n}^{T} W=l_{n}^{T}$ ), and again the left hand side is zero for all $n$. (Such symmetry obtains in (1.4).) Thus if we focus on $\lambda_{0}$ and slope parameters only, their estimates are independent of $\hat{\sigma}^{* 2}$ more generally than Lee (2004) claims, to enhance further the value of his results.

These observations suggest that if we incorporate in our semiparametric likelihood a Jacobian factor $\operatorname{det}\left\{I_{n}-\lambda W\right\}$, the consequent adaptive estimate may achieve both sufficient bias-correction to enable relaxation of (3.1) to (3.13), and the information matrix block-diagonality necessary for adaptivity, when either $W$ is symmetric or $\varepsilon_{i}$ is symmetrically distributed (the moments $E\left(\varepsilon_{i}^{3}\right)$ in the above discussion are replaced by $E\left(\varepsilon_{i} \psi\left(\varepsilon_{i}\right)^{2}\right)$. Essentially, the corresponding term $\operatorname{tr}\left\{W\left(I_{n}-\lambda W\right)^{-1}\right\}$ in $(2.26)$ is of order $n / h$, whence after $n^{-\frac{1}{2}}$ norming a bias of order $n^{\frac{1}{2}} / h$ has been removed, and so Assumption 1 can be relaxed. However, we have not been able to avoid an additional requirement that the series approximation to $\psi(s)$ converges fast enough.

Assumption 7 As $n \rightarrow \infty$

$$
E\left\{\bar{\phi}^{(L)}\left(\varepsilon_{i}\right)^{T} a^{(L)}-\psi\left(\varepsilon_{i}\right)\right\}^{2}=o\left(\frac{h^{2}}{n}\right) .
$$

Under the conditions of Theorem A, the left side of (3.15) is $o(1)$, which suffices there. This situation also obtains in (3.15) in the knife-edge case when $h$ increases like $n^{\frac{1}{2}}$, but when $h=o\left(n^{\frac{1}{2}}\right)$ a rate of convergence is implied by Assumption 7. Since Assumption 5 heavily restricts the increase of $L$ with $n$ the rate implied by (3.15), as a function of $L$, may need to be fast, the more so the slower $h$ increases.

For proof details of the following Theorem see Appendix B.

Theorem B Let (1.2) hold with $\lambda_{0} \in(-1,1)$, and let Assumptions 1-7 hold with (3.1) relaxed to (3.13), and let either $W$ be symmetric or $\varepsilon_{i}$ be symmetrically distributed. Then as $n \rightarrow \infty$,

$$
n^{\frac{1}{2}}\left(\widehat{\theta}_{B}-s\right) \rightarrow_{d} N\left(0, \frac{\sigma_{0}^{2}}{\mathcal{I}} \Omega^{-1}\right)
$$

where the limit variance matrix is consistently estimated by $\left(\tilde{\sigma}^{2} / \tilde{\mathcal{I}}_{L}(\tilde{\theta}, \tilde{\sigma})\right) n R^{-1}$.

Remark 8 In general $\hat{\theta}_{B}$ can be expensive to compute because the second component of $s_{L}(\theta, \sigma)$ involves the inverse of an $n \times n$ matrix. However, in some special cases it is very simple, e.g. in case $W$ is given by (1.4), we have

$$
\operatorname{tr}\left\{W\left(I_{n}-\lambda W\right)^{-1}\right\}=\frac{n \lambda}{(q-1+\lambda)(1-\lambda)} .
$$

Remark 9 We cannot use OLS for $\tilde{\theta}, \tilde{\sigma}^{2}$ if (3.1) does not hold. We can, however, use an IV estimate, such as those of Kelejian and Prucha $(1998,1999)$, Lee (2003), or the Gaussian pseudo-MLE of Lee (2004). 
Remark 10 As in other models, under symmetry of $\varepsilon_{i}$ it is also possible to adapt with respect to the estimation of $\mu_{0}$ in (1.2) (see e.g. Bickel (1982)).

With respect to $\hat{\theta}_{C}$ and $\hat{\theta}_{D}$ we introduce the following additional assumptions.

Assumption 8: $\mathcal{T}$ is compact and $\tau$ is an interior point of $\mathcal{T}$.

Assumption 9: For all $\tau \in \mathcal{T}-\left\{\tau_{0}\right\}, f(s ; \tau) \neq f\left(s ; \tau_{0}\right)$ on a set of positive measure.

Assumption 10: In a neighbourhood $\mathcal{N}$ of $\tau_{0}, \log f(s ; \tau)$ is thrice continuously differentiable in $\tau$ for all $s$ and

$$
\int_{-\infty}^{\infty}\left\{\sup _{\mathcal{N}}\left|f^{(k)}(s ; \tau)\right|+\sup _{\mathcal{N}}\left|f^{(k, \ell)}(s ; \tau)\right|+\sup _{\mathcal{N}}\left|f^{(k, \ell, m)}(s ; \tau)\right|\right\} d s<\infty
$$

where $f^{(k)}, f^{(k, \ell)}, f^{(k, \ell, m)}$ represent partial derivatives of $f$ with respect to the $k$-th, the $k$-th and $\ell$-th, and the $k$-th, $\ell$-th and $m$-th elements of $\tau$, respectively.

Assumption 11: $\Psi=E\left((\partial / \partial \tau) \log f\left(\varepsilon_{i} ; \tau_{0}\right)\left(\partial / \partial \tau^{T}\right) \log f\left(\varepsilon_{i} ; \tau_{0}\right)\right)$ is positive definite.

Theorem C Let (1.2) hold with $\lambda_{0} \in(-1,1)$, and let Assumptions 1-3, 6 and 8-11 hold. Then as $n \rightarrow \infty, n^{\frac{1}{2}}\left(\hat{\theta}_{C}-\theta_{0}\right)$ and $n^{\frac{1}{2}}\left(\widehat{\tau}-\tau_{0}\right)$ converge to independent $N\left(0,\left(\sigma_{0}^{2} / \mathcal{I}\right) \Omega^{-1}\right)$, and $N\left(0, \Psi^{-1}\right)$ vectors respectively, where the limiting covariance matrices are consistently estimated by $\left(\tilde{\sigma}^{2} / \tilde{\mathcal{I}} L(\tilde{\theta}, \tilde{\sigma}, \widehat{\tau})\right) n R^{-1}$ and

$$
\left\{n^{-1} \sum_{t=1}^{n}\left[(\partial / \partial \tau) \log f\left(E_{i}(\tilde{\theta}) / \tilde{\sigma} ; \widehat{\tau}\right)\right]\left[\left(\partial / \partial \tau^{T}\right) \log f\left(E_{i}(\tilde{\theta}) / \tilde{\sigma}_{2} ; \widehat{\tau}\right)\right]\right\}^{-1}
$$

respectively.

Theorem D Let (1.2) hold with $\lambda_{0} \in(-1,1)$, and let Assumptions 13 and 6-11 hold with (3.1) relaxed to (3.13), and let either $W$ be symmetric or $\varepsilon_{i}$ be symmetrically distributed. Then as $n \rightarrow \infty, n^{\frac{1}{2}}\left(\hat{\theta}_{D}-\theta_{0}\right)$ and $n^{\frac{1}{2}}\left(\widehat{\tau}-\tau_{0}\right)$ converge to independent $N\left(0,\left(\sigma_{0}^{2} / \mathcal{I}\right) \Omega^{-1}\right)$, and $N\left(0, \Psi^{-1}\right)$ vectors respectively, where the limiting covariance matrices are consistently estimated by $\left(\tilde{\sigma}^{2} / \tilde{\mathcal{I}} L(\tilde{\theta}, \tilde{\sigma}, \widehat{\tau})\right) n R^{-1}$ and (3.19) respectively. 
The proofs would require first an initial consistency proof for the implicitlydefined extremum estimate $\widehat{\tau}$, and are omitted because they combine elements of the proof of Theorem A with relatively standard arguments.

Remark 11 The Gaussian MLE can in general be expensive to compute due to the determinant factor, as discussed by Kelejian and Prucha (1999). However, the limit distribution of this estimate is the same as that of $\hat{\theta}_{C}$ and $\hat{\theta}_{D}$ when these are based on $f(s ; \tau)=(2 \pi)^{-1 / 2} \exp \left(-s^{2} / 2\right), \psi(s ; \tau)=s$. Indeed such $\hat{\theta}_{D}$ represents a Newton step to the Gaussian MLE.

\section{Finite Sample Performance}

The behaviour of our adaptive estimates in finite sample sizes was examined in a small Monte Carlo study. The spatial weight matrix $W$ given by (1.4) was employed, with three different choices of $(p, q)$ : $(8,12)$, $(11,18),(14,28)$. These correspond to $n=96,198$ and 392, and are intended to represent a slow approach to the asymptotic behaviour of (3.1). For each $n$, scalar explanatory variables $x_{1}, \ldots, x_{n}$ were generated as iid uniform $(0,1)$ observations, and then kept fixed throughout the study, to conform to the non-stochastic aspect of Assumption 2. The $\varepsilon_{i}$ were generated from each of 5 distributions, each of which is presented with the respective asymptotic relative efficiency (ARE) of the Gaussian MLE (i.e. $\mathcal{I}^{-1}$ ).

(a) Normal, $\varepsilon_{i} \sim N(0,1), A R E=1$;

(b) Bimodal Mixture normal, $\varepsilon_{i}=u / \sqrt{10}$ where $p d f(u)=\frac{.5}{\sqrt{2 \pi}} \exp \left(-\frac{(u-3)^{2}}{2}\right)+$ $\frac{.5}{\sqrt{2 \pi}} \exp \left(-\frac{(u+3)^{2}}{2}\right), \quad A R E=0.104 ;$

(c) Unimodal Mixture normal, $\varepsilon_{i}=u / \sqrt{2.2}$, where $p d f(u)=\frac{.05}{\sqrt{50 \pi}} \exp \left(-\frac{u^{2}}{50}\right)+$ $\frac{.95}{\sqrt{2 \pi}} \exp \left(-\frac{u^{2}}{2}\right), \quad A R E=0.514$

(d) Laplace, $f(s)=\exp (-|s| \sqrt{2}) \sqrt{2}, \quad A R E=0.5$;

(e) Student $t_{5}, \varepsilon_{i}=u \sqrt{3 / 5}$, where $u \sim t_{5}, A R E=0.533$.

The ARE was computed by numerical quadrature in cases (b) and (c), and from analytic formulae in the other cases. The distributions (a)-(e) are fairly standard choices in Monte Carlo studies of adaptive estimates. All are scaled to have variance 1, as in Assumption 3. Case (e) has finite moments of degree 4 only.

On each of 1000 replications, $y$ was generated from (1.2) with $\mu_{0}=0, \gamma_{0}=1$, $\beta_{0}=1$, and with two different $\lambda_{0}$ values, 0.4 and 0.8 , for each of the $3 n$ values and $5 \varepsilon_{i}$ distributions. Both $\hat{\theta}_{A}$ and $\hat{\theta}_{B}$ were computed in each case, for both 
choices (2.29) and (2.30) of $\phi(s)$ (respectively denoted "1" and "2" in the tables below), and for $L=1,2,4$. We took $\tilde{\theta}$ to be OLS.

Lee (2004) featured the design (1.4) in his Monte Carlo study of the Gaussian MLE. The two experiments are not closely comparable. He employed a wider range of $n$ and $x_{i}$, while restricting to Gaussian $\varepsilon_{i}$ and a single $\lambda_{0}(0.5)$, and with no comparison with other estimates. Our study looks at relative efficiency over a range of distributions, our examination of two values of $\lambda_{0}$ turns out to throw light on the bias issue, and we explore aspects of implementation which do not arise for his estimate. Nevertheless we shall have occasion to refer to his results, and make some remarks about computational issues prompted by both studies.

Monte Carlo bias, variance and mean squared error (MSE) were computed in each of the $2 \times 2 \times 2 \times 3 \times 3 \times 5=360$ cases. In view of the potential impact of bias, Table 1 reports Monte Carlo bias of both elements, $\widetilde{\lambda}, \widetilde{\beta}$, of the initial estimate, OLS. For $\lambda_{0}=0.4$ the bias of $\widetilde{\lambda}$ actually increases with $n$, suggesting that a faster increase of $q / p$ would give better results here. For $\lambda_{0}=0.8$, biases for the smaller $n$ are greater, they fall then rise with a slight net reduction. We will encounter some consequences of this bias on $\hat{\theta}_{A}$ and $\hat{\theta}_{B}$. The bias of $\widetilde{\beta}$ is much smaller, a phenomenon found also for $\hat{\theta}_{A}$ and $\hat{\theta}_{B}$, and by Lee (2004) for his estimate.

(Table 1 about here)

Tables 2-5 respectively present relative variance of $\widehat{\lambda}_{A}$, relative MSE of $\widehat{\lambda}_{A}$, relative variance of $\widehat{\beta}_{A}$, and relative MSE of $\widehat{\beta}_{A}$, in each case comparing the appropriate element of $\hat{\theta}=\left(\widehat{\lambda}_{A}, \widehat{\beta}_{A}\right)^{T}$ with OLS for each of the distributions (b)-(e), covering all $n, \lambda_{0}, L$ and $\phi$. To conserve on space we have omitted the normal distribution (a) results. Here, one expects $\hat{\theta}_{A}$ to be worse than OLS for all $L \geq 1$ when $\phi=(2.30)$, and to deteriorate with increasing $L$ when $\phi=$ (2.29). This turned out to be the case, but though the ratios peaked at 1.4447 (in case of relative variance of $\widehat{\lambda}_{A}$ for $\lambda_{0}=0.8, n=96, L=2, \phi=(2.30)$ ), they were mostly less than 1.1. Note that for the other distributions the ratios of 1 when $\phi=(2.29)$ and $L=1$ reflect the identity $\hat{\theta}_{A}=\widetilde{\theta}$.

For the bimodal mixture normal distribution (b), though $\hat{\theta}_{A}$ is sometimes worse than $\widetilde{\theta}$ for small $L$, by $L=4$ a clear, sometimes dramatic improvement was registered, especially in the MSE ratios, with the ARE being well approximated in case of $\widehat{\beta}_{A}$. As the ARE calculations indicate, distribution (b) has the greatest potential for efficiency improvement. Our Monte Carlo results for distributions (a)-(e) reflect this, though ARE is nearly always over-estimated, in some cases considerably. Nevertheless, except for $\lambda_{0}=0.8$ (in relative variance Table 2), $\hat{\theta}_{A}$ always beats OLS, to varying degrees. Some summary statistics based on Tables 2-5 are useful. Consider first the property of monotone improvement with increasing $n$ or $L$ (we do not count cases when, say, there is ultimate improvement without monotonicity). There is monotone improvement with increasing $n$ in 84 (30 for $\widehat{\lambda}_{A}, 54$ for $\widehat{\beta}_{A}$ ) out of 160 places, with distribution (c) best and (a) worst. There is monotone improvement with increasing $L$ in 104 (48 for $\widehat{\lambda}_{A}, 56$ for $\widehat{\beta}_{A}$ ) out of 196 places, with (b) best and (d) worst. In both 
instances, the number of such improvements was somewhat greater for $\lambda_{0}=0.4$ than $\lambda_{0}=0.8$. With respect to choice of $\phi$, there is monotone improvement with increasing $n$ in 23 of 64 places for (2.29) (omitting $L=1$ of course) and 62 of 96 for $(2.30)$.

(Tables 2-5 about here)

The disappointing aspects of Table 2 serve as a prelude to the results for $\hat{\theta}_{B}=$ $\left(\widehat{\lambda}_{B}, \widehat{\beta}_{B}\right)^{T}$ when $\lambda_{0}=0.8$. What happens is that the second ("bias correction") component in $s_{L}$ vastly overcompensates for the positive bias in $\tilde{\lambda}$ seen in Table 1. The reason is apparent from (3.17). Overestimation of $\lambda_{0}$ not only increases the numerator but brings the denominator close to zero. In one place $\widehat{\lambda}_{B}$ beats OLS, and $\widehat{\beta}_{A}$ does so in 46 , but these are out of 144 in each case, and overall the results are too poor to report. However, we present the results for $\lambda_{0}=$ 0.4 , in Tables 6 and 7, combining relative variance and MSE in each table. Of most interest is comparison of $\hat{\theta}_{B}$ with $\hat{\theta}_{A}$. Of the 288 places, $\hat{\theta}_{B}$ does best in 124; 93 of these are relative variances, and 70 refer to $\widehat{\beta}_{B}$. The bias-correction is not very successful even when $\lambda_{0}=0.4$, with $\widetilde{\lambda}$ still largely to blame. There is monotone improvement with increasing $n$ in 23 ( 11 for $\widehat{\lambda}_{B}, 12$ for $\widehat{\beta}_{B}$ ) out of 96 places, with distribution (c) best (again) and (e) worst, so $\hat{\theta}_{B}$ performs worse than $\hat{\theta}_{A}$ in this respect also. On the other hand, there is monotone improvement with increasing $L$ in 56 (28 each for $\widehat{\lambda}_{B}$ and $\widehat{\beta}_{B}$ ) out of 92 places, with (b) best (again) and the others roughly equal. Again the choice (2.30) of $\phi$ fares better than (2.29) with respect to monotone improvement with increasing $n, 16$ to 7 .

(Tables 6-7 about here)

Clearly $\hat{\theta}_{D}$, in particular a Newton approximation to the Gaussian MLE, will be similarly affected, relative to $\hat{\theta}_{C}$. Lee (2004), in his Monte Carlo, used a search algorithm to compute the Gaussian MLE itself, thereby not risking contamination by an initial estimate. However, the larger $n$, and especially $k$, the more expensive this approach becomes, and it could prove prohibitive, especially when $W$ leads to a less tractable $\operatorname{det}\left\{I_{n}-\lambda W\right\}$ than is the case with (1.4) (see Kelejian and Prucha (1999). Iteration from an initial estimate may then be preferable (which brings us back to $\hat{\theta}_{D}$ ). On the other hand, the present paper has stressed ahievement of asymptotic efficiency in a general setting, with a minimum of computation. In a given practical situation, this may not be the most relevant goal, and improvements might be desirable, perhaps especially to $\hat{\theta}_{B}$ and $\hat{\theta}_{D}$, by exercizing greater care in choice of $\widetilde{\theta}$ (possibly using one of the instrumental variables estimates in the literature), and continuing the iterations. This will incur greater computational expense, though updating of $R$ does not arise. These and other issues might be examined in a subsequent, more thorough, Monte Carlo study. It is hoped that the present simulations have demonstrated that the computationally simple estimates $\hat{\theta}_{A}$ and $\hat{\theta}_{B}$, with their optimal asymptotic properties in a wide setting, offer sufficient promise to warrant such investigation and possible refinement, and empirical application. 


\section{APPENDIX A: Proof of Theorem A}

By the mean value theorem

$$
\begin{aligned}
& \hat{\theta}_{A}-\theta_{0}=\left(I_{k+1}-\frac{R^{-1}}{\tilde{\mathcal{I}}_{L}(\tilde{\theta}, \tilde{\sigma})} \bar{S}_{1 L}\right)\left(\tilde{\theta}-\theta_{0}\right) \\
& -\frac{R^{-1}}{\tilde{\mathcal{I}}_{L}(\tilde{\theta}, \tilde{\sigma})}\left\{\bar{S}_{2 L}\left(\tilde{\sigma}-\sigma_{0}\right)+r_{L}\left(\theta_{0}, \sigma_{0}\right)\right\}
\end{aligned}
$$

where $\bar{S}_{1 L}$ and $\bar{S}_{2 L}$ are respectively obtained from $S_{1 L}(\theta, \sigma)=\left(\partial / \partial \theta^{T}\right) r_{L}(\theta, \sigma)$ and $S_{2 L}(\theta, \sigma)=(\partial / \partial \sigma) r_{L}(\theta, \sigma)$ after evaluating each row at some (possibly different) $\bar{\theta}, \bar{\sigma}$ such that $\left\|\bar{\theta}-\theta_{0}\right\| \leq\left\|\tilde{\theta}-\theta_{0}\right\|,\left|\bar{\sigma}-\sigma_{0}\right| \leq\left|\tilde{\sigma}-\sigma_{0}\right|$. Introduce the neighbourhood $\mathcal{N}=\left\{\theta, \sigma:\left\|\theta-\theta_{0}\right\|+\left\|\sigma-\sigma_{0}\right\| \leq n^{-\frac{1}{2}}\right\}$. In view of Assumptions 2 and 3 , the proof consists of showing that

$$
\begin{aligned}
\sup _{\mathcal{N}}\left\|S_{i L}(\theta, \sigma)-S_{i L}\left(\theta_{0}, \sigma_{0}\right)\right\|=o_{p}(n), n=1,2, & \\
& \sup _{\mathcal{N}}\left|\tilde{\mathcal{I}}_{L}(\theta, \sigma)-\mathcal{I}_{L}\left(\theta_{0}, \sigma_{0}\right)\right| \rightarrow_{p} 0, \\
& n^{-1} R \rightarrow p \Omega \\
& {\left[\tilde{\mathcal{I}}_{L}\left(\theta_{0}, \sigma_{0}\right) R\right]^{-1} S_{1 L}\left(\theta_{0}, \sigma_{0}\right) \rightarrow{ }_{p} I_{k+1}, } \\
& n^{-1} S_{L 2}\left(\theta_{0}, \sigma_{0}\right) \rightarrow p 0, \\
& \tilde{\mathcal{I}}_{L}\left(\theta_{0}, \sigma_{0}\right) \rightarrow p \mathcal{I}, \\
r_{1 L}= & o_{p}\left(n^{\frac{1}{2}}\right), \\
& n^{-\frac{1}{2}} r_{2 L} \rightarrow{ }_{d} \mathcal{N}\left(0, \sigma_{0} \mathcal{I} \Omega\right),
\end{aligned}
$$

where

$$
r_{j L}=\sum_{i} \tilde{\psi}_{i L}\left(\theta_{0}, \sigma_{0}\right) E_{j i}^{\prime}, \quad j=1,2,
$$

in which $\sum_{i}$ denotes $\sum_{i=1}^{n}$,

$$
\begin{aligned}
& \left(E_{11}^{\prime}, \ldots, E_{1 n}^{\prime}\right)=E_{1}^{\prime}=-\sigma_{0}(H G \varepsilon, 0)^{T}, \\
& \left(E_{21}^{\prime}, \ldots, E_{2 n}^{\prime}\right)=E_{2}^{\prime}=-\left(H G X \beta_{0}, H X\right)^{T} .
\end{aligned}
$$

Notice that $r_{L}\left(\theta_{0}, \sigma_{0}\right)=r_{1 L}+r_{2 L}$, due to $E^{\prime}=H e^{\prime}=E_{1}^{\prime}+E_{2}^{\prime}$, since

$$
\begin{aligned}
e^{\prime} & =-\left(G\left(l_{n} \mu_{0}+X \beta_{0}+\sigma_{0} \varepsilon\right), X\right)^{T} \\
& =-\left(\left(1-\lambda_{0}\right)^{-1} l_{n} \mu_{0}+G\left(X \beta_{0}+\sigma_{0} \varepsilon\right), X\right)^{T} .
\end{aligned}
$$


The proof of (A.9) is essentially as in Newey (1988, Theorem 2.3), Robinson (2005, Theorem 1) (the weaker conditions in the latter reference being reflected in Assumption 5). The only difference is the triangular array structure in the first element of $r_{2 L}$. This makes no real difference to the proof that the $\tilde{\psi}_{i L}\left(\theta_{0}, \sigma_{0}\right)$ can be replaced by the $\psi\left(\varepsilon_{i}\right)$, whence $n^{-\frac{1}{2}} \sum_{i} \psi\left(\varepsilon_{i}\right) E_{2 i}^{\prime} \rightarrow_{d}$ $N\left(0, \sigma_{0}^{2} \mathcal{I} \Omega\right)$ follows from a triangular-array central limit theorem (such as Lemma A.2 of Lee, 2002).

To prove (A.8), write

$$
\begin{aligned}
r_{1 L} & =\sigma_{0}\left(a_{1}+a_{2}+a_{3}+a_{4}, 0\right)^{T} \\
a_{j} & =\sum_{i} b_{j i} \chi_{i n}, \quad j=1, \ldots, 4, \\
\chi_{i n} & =\varepsilon^{T} G^{T}\left(1_{i}-l_{n} / n\right), \\
b_{1 i} & =\psi\left(\varepsilon_{i}\right), \\
b_{2 i} & =\bar{\psi}^{(L)}\left(\varepsilon_{i} ; a^{(L)}\right)-\psi\left(\varepsilon_{i}\right), \\
b_{3 i} & =\psi^{(L)}\left(\varepsilon_{i} ; \tilde{a}^{(L)}(\varepsilon)\right)-\bar{\psi}^{(L)}\left(\varepsilon_{i} ; a^{(L)}\right), \\
b_{4 i} & =\tilde{\psi}_{i}^{(L)}\left(\theta_{0}, \sigma_{0}\right)-\psi^{(L)}\left(\varepsilon_{i} ; \tilde{a}^{(L)}(\varepsilon)\right),
\end{aligned}
$$

in which $\bar{\psi}^{(L)}\left(\varepsilon_{i} ; a^{(L)}\right)=\bar{\phi}^{(L)}(\varepsilon)^{T} a^{(L)}$ (cf. (2.3)) and $1_{i}$ is the ith column of $I_{n}$

Define

$$
t_{i j n}=1_{j}^{T} G^{T}\left(1_{i}-l_{n} / n\right)=1_{j}^{T} G^{T} 1_{i}-\sum_{\ell} 1_{j}^{T} G^{T} 1_{\ell} / n,
$$

so that

$$
\chi_{i n}=\sum_{j} \varepsilon_{j} t_{i j n}
$$

Thus write

$$
a_{1}=\sum_{i} \psi\left(\varepsilon_{i}\right) \varepsilon_{i} t_{i i n}+\sum_{i} \psi\left(\varepsilon_{i}\right) \sum_{j \neq i} \varepsilon_{j} t_{i j n}
$$

We have

$$
\left|t_{i j n}\right| \leq\left|1_{j}^{T} G^{T} 1_{i}\right|+\sum_{\ell}\left|1_{j}^{T} G^{T} 1_{\ell}\right| / n .
$$

For all $i, j$, Assumption 1 implies

$$
1_{i}^{T} G^{T} 1_{j}=1_{j}^{T} W S^{-1} 1_{i}=O\left(h^{-1}\right)
$$

uniformly. (Lee $\left(2002\right.$, p.258) gives (A.25) for $i=j$.) It follows that $t_{i j n}$ $=O_{p}\left(h^{-1}\right)$ uniformly. Then the absolute value of the first term on the right of (A.23) has expectation bounded by $\left\{E \psi^{2}\left(\varepsilon_{i}\right) E \varepsilon_{i}^{2}\right\}^{\frac{1}{2}} \sum_{i}\left|t_{i i n}\right|=O_{p}(n / h)$, by the Schwarz inequality. The second term in (A.23) has mean zero and variance $O(n / h)$. The proof of the latter statement is quickly obtained from that of Lemma A.1 of Lee (2002), which covers $\sum_{i} \sum_{j} \varepsilon_{i} \varepsilon_{j} t_{i j n}$ : we can replace $\varepsilon_{i}$ by $\psi\left(\varepsilon_{i}\right)$, noting

$$
E\left(\varepsilon_{i} \psi\left(\varepsilon_{i}\right)\right)=-\int s f^{\prime}(s) d s=E\left(\varepsilon_{i}^{2}\right)
$$


(by integration-by-parts), as well as Assumption 3, and we omit "diagonal terms" $i=j$ of Lee's (2002) statistic to negligible effect, thus implying that we do not require $E\left(\varepsilon_{i}^{2} \psi\left(\varepsilon_{i}\right)^{2}\right)<\infty$, which would correspond to his condition $E\left(\varepsilon_{i}^{4}\right)<\infty$. It follows that $a_{1}=O_{p}\left(n / h+(n / h)^{\frac{1}{2}}\right)=o_{p}\left(n^{\frac{1}{2}}\right)$.

Next write

$$
a_{2}=\sum_{i} \tau_{i}^{(L)} \varepsilon_{i} t_{i i n}+\sum_{i} \tau_{i}^{(L)} \sum_{j \neq i} \varepsilon_{j} t_{i j n},
$$

where $\tau_{i}^{(L)}=\bar{\psi}^{(L)}\left(\varepsilon_{i} ; a^{(L)}\right)-\psi\left(\varepsilon_{i}\right)$. The absolute value of the first term has expectation bounded by

$$
\left\{E\left(\tau_{1}^{(L) 2}\right)\right\}^{\frac{1}{2}} \sum_{i}\left|t_{i i n}\right|,
$$

again using the Schwarz inequality. The expectation in (A.28) remains finite as $L \rightarrow \infty$, indeed it tends to zero (see Freud, 1971, pp.77-79), as is crucially used in the proof of (A.9) (see Newey $(1988$, p.329)). Thus (A.28) $=o(n / h)$. From Assumption 3, the second term of (A.27) has mean zero and variance

$$
\sum_{i} E\left(\tau_{i}^{(L) 2}\right) E\left(\sum_{j \neq i} \varepsilon_{j} t_{j i n}\right)^{2}+\sum_{i} \sum_{j} E\left(\tau_{i}^{(L)} \varepsilon_{i}\right) E\left(\tau_{j}^{(L)} \varepsilon_{j}\right) t_{j i n} t_{i j n} .
$$

The first term is $o\left(\sum_{i} \sum_{j} t_{j i n}^{2}\right)=o\left(n^{2} / h^{2}\right)$, while the second is

$$
O\left(\sum_{i} E\left(\tau_{i}^{(L) 2}\right) E \varepsilon_{i}^{2} \sum_{j} t_{j i n}^{2}\right)=o\left(\sum_{i} \sum_{j} t_{j i n}^{2}\right)=o\left(n^{2} / h^{2}\right)
$$

also. We have shown that $E\left(a_{2}^{2}\right)=o\left(n^{2} / h^{2}\right)$, whence $a_{2}=o_{p}\left(n^{\frac{1}{2}}\right)$ from Assumption 1.

Since $\sum_{i} t_{i j n}=0$ for all fixed $j$, we have $\sum_{i} \chi_{i n}=0$, and thence

$$
a_{3}=\left\{\tilde{a}^{(L)}(\varepsilon)-a^{(L)}\right\}^{T} \sum_{i} \bar{\phi}^{(L)}\left(\varepsilon_{i}\right) \chi_{i n} .
$$

Proceeding as before, write

$$
\sum_{i} \bar{\phi}^{(L)}\left(\varepsilon_{i}\right) \chi_{i n}=\sum_{i} \bar{\phi}^{(L)}\left(\varepsilon_{i}\right) \varepsilon_{i} t_{i i n}+\sum_{j \neq i} \bar{\phi}^{(L)}\left(\varepsilon_{i}\right) \sum_{j \neq i} \varepsilon_{j} t_{i j n} .
$$

As in Robinson (2005), introduce the notation

$$
\mu_{c}=1+E\left|\varepsilon_{i}\right|^{c}, \quad c \geq 0,
$$

and

$$
\begin{aligned}
& \rho_{u L}=C L, \quad \text { if } u=0, \\
= & (C L)^{u L / \omega}, \quad \text { if } u>0 \text { and Assumption 5(ii) holds, } \\
= & C^{L}, \quad \text { if } u>0 \text { and Assumption 5(iii) holds, }
\end{aligned}
$$


suppressing reference to $C$ in $\rho_{u L}$. With $\|$.$\| denoting (Euclidean) norm, the$ norm of the first term on the right of (A.32) has expectation bounded by

$$
\begin{aligned}
\sum_{i}\left\{E\left\|\bar{\phi}^{(L)}\left(\varepsilon_{i}\right)\right\|^{2}\right\}^{\frac{1}{2}} t_{i i n}^{2} & \leq \frac{C n}{h}\left\{\sum_{\ell=1}^{L} E \phi^{2 \ell}\left(\varepsilon_{i}\right)\right\}^{\frac{1}{2}} \\
& \leq \frac{C n}{h}\left\{\sum_{\ell=1}^{L} \mu_{2 \kappa L}\right\}^{\frac{1}{2}} \\
& \leq \frac{C n}{h} \rho_{2 \kappa L}^{\frac{1}{2}},
\end{aligned}
$$

using Assumption 4, and then Lemma 9 of Robinson (2005). The second term of (A.32) has zero mean vector and covariance matrix

$$
\begin{aligned}
& \sum_{i} E\left\{\bar{\phi}^{(L)}\left(\varepsilon_{i}\right) \bar{\phi}^{(L)}\left(\varepsilon_{i}\right)^{T}\right\} \sum_{j \neq i} t_{i j n}^{2} \\
& +\sum_{i} \sum_{j} E\left\{\bar{\phi}^{(L)}\left(\varepsilon_{i}\right) \varepsilon_{i}\right\} E\left\{\bar{\phi}^{(L)}\left(\varepsilon_{j}\right) \varepsilon_{j}\right\}^{T} t_{i j n} t_{j i n},
\end{aligned}
$$

which from earlier calculations has norm $O\left(n^{2} \rho_{2 \kappa L} / h^{2}\right)$. Thus

$$
\left\|\sum_{i} \bar{\phi}^{(L)}\left(\varepsilon_{i}\right) \chi_{i n}\right\|=O_{p}\left(\frac{n \rho_{2 \kappa L}^{\frac{1}{2}}}{h}\right) .
$$

It follows from Lemma 10 of Robinson (2005) that

$$
a_{3}=O_{p}\left(\frac{n^{\frac{1}{2}}}{h} L^{3 / 2} \rho_{2 \kappa L} \rho_{4 \kappa L}^{\frac{1}{2}} \pi_{L}^{2}\right)
$$

where

$$
\pi_{L}=(\log L) \eta^{2 L} 1(\varphi<1)+(L \log L) \eta^{2 L} 1(\varphi=1)+(\log L)(\eta \varphi)^{2 L} 1(\varphi>1) .
$$

Suppose first Assumption 5(i) holds. It follows from (A.34) and (A.40) that

$$
a_{3}=O_{p}\left(\frac{n^{\frac{1}{2}}}{h} L^{3} \pi_{L}^{2}\right)=O_{p}\left(n^{\frac{1}{2}} \exp \left[\log h\left\{\frac{3 \log L+2 \log \pi_{L}}{\log h}-1\right\}\right]\right),
$$

which is $o_{p}\left(n^{\frac{1}{2}}\right)$ if $\lim \inf \left(\log h / \log \pi_{L}\right)>\frac{1}{2}$, as is true under (3.7). Now suppose Assumption 5(ii) holds. It follows from (A.35) that

$$
a_{3}=O_{p}\left(\frac{n^{\frac{1}{2}}}{h} L^{3 / 2+4 \kappa L / \omega} \pi_{L}^{2}\right)
$$

which is $o_{p}\left(n^{\frac{1}{2}}\right)$ if $\liminf (\log h / L \log L)>4 \kappa / \omega$, as is true under (3.8). Finally, suppose Assumption 5(iii) holds. It follows from (A.36) that

$$
a_{3}=O_{p}\left(\frac{n^{\frac{1}{2}}}{h} L^{3 / 2} C^{L} \pi_{L}^{2}\right)
$$


which is $o_{o}\left(n^{\frac{1}{2}}\right)$ if $(\log h) / L \rightarrow \infty$, as is implied by (3.9). This completes the proof that $a_{3}=o_{p}\left(n^{\frac{1}{2}}\right)$.

Now write

$$
\begin{aligned}
a_{4}= & \left\{\tilde{a}^{(L)}\left(E / \sigma_{0}\right)-\tilde{a}^{(L)}(\varepsilon)\right\}^{T} \sum_{i} \Phi^{(L)}\left(\varepsilon_{i}\right) \chi_{i n} \\
& +\tilde{a}^{(L)}\left(E / \sigma_{0}\right)^{T} \sum_{i}\left\{\Phi^{(L)}\left(E_{i} / \sigma_{0}\right)-\Phi^{(L)}\left(\varepsilon_{i}\right)\right\} \chi_{i n} .
\end{aligned}
$$

By the mean value theorem, with $\bar{\varepsilon}=n^{-1} \sum_{i} \varepsilon_{i}$,

$$
\phi_{\ell}\left(E_{i} / \sigma_{0}\right)-\phi_{\ell}\left(\varepsilon_{i}\right)=-\bar{\varepsilon} \phi_{\ell}^{\prime}\left(\varepsilon_{i}\right)+\frac{1}{2} \bar{\varepsilon}^{2} \phi_{\ell}^{\prime \prime}\left(\varepsilon_{i}^{*}\right)
$$

where $\left|\varepsilon_{i}^{*}-\varepsilon_{i}\right| \leq\left|E_{i} / \sigma_{0}-\varepsilon_{i}\right|=|\bar{\varepsilon}|$. Now

$$
\sum_{i} \phi_{\ell}^{\prime}\left(\varepsilon_{i}\right) \chi_{i n}=\sum_{i}\left\{\phi_{\ell}^{\prime}\left(\varepsilon_{i}\right)-E \phi_{\ell}^{\prime}\left(\varepsilon_{i}\right)\right\} \chi_{i n}
$$

using $\sum_{i} x_{i n}=0$ again. Proceeding much as before, and from Assumption 3, and (6.23) of Robinson (2005), this is $O_{p}\left(\left\{E \phi_{\ell}^{\prime}\left(\varepsilon_{i}\right)^{2}\right\}^{\frac{1}{2}} n / h\right)=O_{p}\left(\ell \mu_{2 \kappa(\ell+K)}^{\frac{1}{2}} n / h\right)$. Using $\left|\varepsilon_{i}^{*}\right| \leq\left|\varepsilon_{i}\right|+|\bar{\varepsilon}|$ and the $c_{r}$-inequality, and proceeding as in Robinson (2005, p. 1822),

$$
\left|\sum_{i} \phi_{\ell}^{\prime \prime}\left(\varepsilon_{i}^{*}\right) \chi_{i n}\right| \leq C^{\kappa \ell+1} \ell^{2} \sum_{i}\left\{1+\left|\varepsilon_{i}\right|^{\kappa(\ell-1+2 K)}+|\bar{\varepsilon}|^{\kappa(\ell-1+2 K)}\right\}\left|\chi_{i n}\right| .
$$

The Schwarz inequality gives

$$
\begin{aligned}
E\left|\chi_{i n}\right| & \leq\left(E \chi_{i n}^{2}\right)^{1 / 2} \leq\left(\sum_{j} t_{i j n}^{2}\right)^{1 / 2} \leq C n^{1 / 2} / h \\
E\left(\left|\varepsilon_{i}\right|^{\kappa(\ell-1+2 K)}\left|\chi_{i n}\right|\right) & \leq C \mu_{2 \kappa(\ell-1+2 K)}^{1 / 2} n^{1 / 2} / h
\end{aligned}
$$

uniformly in $i$. From Lemma 9 of Robinson (2005) and the Schwarz inequality,

$$
\begin{aligned}
\sum_{\ell=1}^{L} \ell \mu_{2 \kappa(\ell+K)}^{\frac{1}{2}} & \leq C L^{3 / 2} \rho_{2 \kappa L}^{\frac{1}{2}}, \\
\sum_{\ell=1}^{L} C^{\kappa \ell+1} \ell^{2}\left(1+\mu_{2 \kappa(\ell-1+2 K)}^{\frac{1}{2}}\right) & \leq C^{\kappa L+1} L^{3 / 2} \rho_{2 \kappa L}^{\frac{1}{2}} .
\end{aligned}
$$

With $\bar{\varepsilon}=O_{p}\left(n^{-1 / 2}\right)$, we deduce

$$
\left\|\sum_{i}\left\{\Phi^{(L)}\left(E_{i} / \sigma_{0}\right)-\Phi^{(L)}\left(\varepsilon_{i}\right)\right\} \chi_{i n}\right\|=O_{p}\left(C^{\kappa L} L^{5 / 2} \rho_{2 \kappa L}^{\frac{1}{2}} n^{\frac{1}{2}} / h\right) .
$$

From Lemmas 10 and 19 of Robinson (2005), the second term on the right of (A.45) is

$$
O_{p}\left(\frac{n^{\frac{1}{2}}}{h} C^{\kappa L} \rho_{2 \kappa L} L^{7 / 2} \pi_{L}\right) .
$$


Next note that

$$
\sum_{i} \Phi^{(L)}\left(\varepsilon_{i}\right) \chi_{i n}=\sum_{i} \bar{\phi}^{(L)}\left(\varepsilon_{i}\right) \chi_{i n}=O_{p}\left(\frac{n}{h} \rho_{2 \kappa L}^{\frac{1}{2}}\right)
$$

using $\sum_{i} \chi_{i n}=0$ again and (A.39). Thus from Lemma 19 of Robinson (2005), the first term on the right of (A.45) is

$$
O_{p}\left(\frac{n^{\frac{1}{2}}}{h} \rho_{2 \kappa L}^{2} \pi_{L}^{2}\left(L^{2}+(C L)^{4 \kappa L+3} n^{-\frac{1}{2}} \log n\right)\right) .
$$

Suppose Assumption 5(ii) holds. It follows from (A.54) and (A.56) that

$$
\begin{aligned}
a_{4} & =O_{p}\left(\frac{n^{\frac{1}{2}}}{h}\left(L^{11 / 2} \pi_{L}+L^{4} \pi_{L}^{2}+L^{5} \pi_{L}^{2} n^{-\frac{1}{2}} \log n\right)\right) \\
& =O_{p}\left(\frac{n^{\frac{1}{2}}}{h} L^{4} \pi_{L}^{2}\right)=o_{p}\left(n^{\frac{1}{2}}\right)
\end{aligned}
$$

under (3.7). If Assumption 5(ii) holds,

$$
\begin{aligned}
a_{4}= & O_{p}\left(\frac { n ^ { \frac { 1 } { 2 } } } { h } \left\{C^{\kappa L}(C L)^{2 \kappa L / \omega} L^{7 / 2} \pi_{L}\right.\right. \\
& \left.\left.+(C L)^{4 \kappa L / \omega} L^{2} \pi_{L}^{2}+(C L)^{4 \kappa L(1+1 / \omega)} L^{3} \pi_{L}^{2} n^{-\frac{1}{2}} \log n\right\}\right) \\
= & O_{p}\left(\frac{n^{\frac{1}{2}}}{h}\left\{(C L)^{4 \kappa L / \omega}+(C L)^{4 \kappa L(1+1 / \omega)} n^{-\frac{1}{2}} \log n\right\}\right)
\end{aligned}
$$

and this is $o_{p}\left(n^{\frac{1}{2}}\right)$ under (3.8) (the equality in (3.8) being used for the second term and the fact that $4 \kappa(\omega+1) / \omega>4 \kappa / \omega$ used for the first). Finally, under Assumption 5(iii),

$$
\begin{aligned}
a_{4} & =O_{p}\left(\frac{n^{\frac{1}{2}}}{h}\left\{C^{\kappa L} L^{7 / 2} \pi_{L}+C^{\kappa L} L^{2} \pi_{L}^{2}+(C L)^{4 \kappa L} L^{3} \pi_{L}^{2} n^{-\frac{1}{2}} \log n\right\}\right) \\
& =O_{p}\left(\frac{n^{\frac{1}{2}}}{h}\left\{C^{\kappa L}+(C L)^{4 \kappa L} n^{-\frac{1}{2}} \log n\right\}\right) .
\end{aligned}
$$

The second term is $o_{p}\left(n^{\frac{1}{2}}\right)$ under (3.9) (using the equality) and since (3.9) implies $L / \log h \rightarrow \infty$, so is the first.

This completes the proof of (A.8), which is by far the most difficult and distinctive part of the Theorem proofs, due both to the simultaneity problem and the $n^{\frac{1}{2}}$ normalization. We thus omit the proof of (A.2)-(A.7), of which indeed (A.4) is in Lee (2002). 


\section{APPENDIX B: Proof of Theorem B}

We redefine

$$
\begin{aligned}
a_{1}= & \sum_{i}\left\{\psi\left(\varepsilon_{i}\right) \varepsilon_{i}-1\right\} t_{i i n} \\
& -\ell_{n}^{T} G \ell_{n} / n \\
& +\sum_{i} \psi\left(\varepsilon_{i}\right) \sum_{j \neq i} \varepsilon_{j} t_{i j n}
\end{aligned}
$$

(cf. (A.23)). Now (B.1) has mean zero and variance $\sum_{i} t_{i i n}^{2}=O\left(n / h^{2}\right)$, so that $(\mathrm{B} .1)=O_{p}\left(n^{\frac{1}{2}} / h\right)=o_{p}\left(n^{\frac{1}{2}}\right)$. Next $(\mathrm{B} \cdot 2)=-\left(1-\lambda_{0}\right)^{-1}=o_{p}\left(n^{\frac{1}{2}}\right)$. Finally we showed in the proof of Theorem A that $(\mathrm{B} \cdot 3)=O_{p}\left(n^{\frac{1}{2}} / h^{\frac{1}{2}}\right)=o_{p}\left(n^{\frac{1}{2}}\right)$. Thus $a_{1}=o_{p}\left(n^{\frac{1}{2}}\right)$.

Now consider $a_{2}$, defined as in (A.15), (A.27). From Assumption 7, (A.28) $=o\left(m^{\frac{1}{2}}\right)$ and $(\mathrm{A} .19)=o\left(\left(h^{2} / n\right)\left(n^{2} / h^{2}\right)\right)=o(n)$ to verify that $a_{2}=o_{p}\left(n^{\frac{1}{2}}\right)$.

The remainder of the proof of Theorem A applies, since it does not use (3.1) but rather (3.13), as well as Assumption 5, which is expressed in terms of $h$ rather than $n$.

\section{Acknowledgements}

I thank Afonso Goncalves da Silva for carrying out the simulations reported in Section 4, and two referees whose comments have led to improved presentation. This research was supported by ESRC Grants R000239936 and RES-062$23-0036$. 
Table 1: Monte Carlo Bias of OLS estimates $\widetilde{\lambda}, \widetilde{\beta}$.

\begin{tabular}{|c|c|c|c|c|c|c|c|}
\hline & $\begin{array}{l}\lambda_{0} \\
n\end{array}$ & 96 & $\begin{array}{c}0.4 \\
198\end{array}$ & 392 & 96 & $\begin{array}{c}0.8 \\
198\end{array}$ & 392 \\
\hline \multirow{5}{*}{$\widetilde{\lambda}$} & (a) & 0.0436 & 0.0995 & 0.1397 & 0.1373 & 0.1289 & 0.1376 \\
\hline & (b) & 0.0704 & 0.1029 & 0.1336 & 0.1399 & 0.1296 & 0.1362 \\
\hline & (c) & 0.0743 & 0.1125 & 0.1384 & 0.1410 & 0.1297 & 0.1364 \\
\hline & (d) & 0.0414 & 0.1102 & 0.1337 & 0.1370 & 0.1305 & 0.1365 \\
\hline & (e) & 0.0738 & 0.1056 & 0.1337 & 0.1411 & 0.1295 & 0.1365 \\
\hline \multirow{5}{*}{$\widetilde{\beta}$} & (a) & 0.0103 & -0.0114 & -0.0125 & 0.0155 & -0.0434 & -0.0291 \\
\hline & (b) & -0.0033 & -0.0126 & -0.0145 & -0.0007 & -0.0447 & -0.0324 \\
\hline & (c) & -0.0058 & -0.0061 & 0.0011 & -0.0029 & -0.0381 & -0.0163 \\
\hline & (d) & 0.0041 & -0.0147 & -0.0151 & 0.0095 & -0.0462 & -0.0321 \\
\hline & (e) & 0.0067 & 0.0036 & -0.0074 & 0.0091 & -0.0272 & -0.0243 \\
\hline
\end{tabular}

Table 2: Monte Carlo Relative Variance, $\operatorname{Var}\left(\widehat{\lambda}_{A}\right) / \operatorname{Var}(O L S)$.

\begin{tabular}{|c|c|c|c|c|c|c|c|c|}
\hline & $\phi$ & $\begin{array}{r}\lambda_{0} \\
L \backslash n\end{array}$ & 96 & $\begin{array}{c}0.4 \\
198\end{array}$ & 392 & 96 & $\begin{array}{c}0.8 \\
198\end{array}$ & 392 \\
\hline \multirow{6}{*}{ (b) } & \multirow{3}{*}{1} & 1 & 1.0000 & 1.0000 & 1.0000 & 1.0000 & 1.0000 & $\bar{~} 1.0000$ \\
\hline & & 2 & 1.0116 & 0.9978 & 0.9712 & 1.0499 & 1.1077 & 1.0492 \\
\hline & & 4 & 0.3165 & 0.1395 & 0.1406 & 0.4688 & 0.5954 & 0.7249 \\
\hline & \multirow{3}{*}{2} & 1 & 1.8846 & 2.3580 & 2.4790 & 2.1239 & 2.2482 & 2.3935 \\
\hline & & 2 & 1.4788 & 2.0466 & 2.0889 & 2.9373 & 4.3919 & 4.5746 \\
\hline & & 4 & 0.2809 & 0.0894 & 0.0972 & 0.3306 & 0.4559 & 0.5405 \\
\hline \multirow{6}{*}{ (c) } & \multirow{3}{*}{1} & 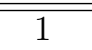 & $\overline{c 1.0000}$ & $\overline{c 1.0000}$ & " 1.0000 & $\bar{~} 1.0000$ & $\overline{c 1.0000}$ & $\overline{c 1.0000}$ \\
\hline & & 2 & 0.8428 & 0.8339 & 0.9142 & 1.6047 & 1.5192 & 1.4857 \\
\hline & & 4 & 0.5876 & 0.5565 & 0.5257 & 1.5906 & 1.3757 & 1.4045 \\
\hline & \multirow{3}{*}{2} & 1 & 0.6517 & 0.5925 & 0.5392 & 1.2441 & 1.1066 & 1.0893 \\
\hline & & 2 & 0.6763 & 0.5873 & 0.5417 & 1.4211 & 1.1593 & 1.1204 \\
\hline & & 4 & 0.6813 & 0.6088 & 0.5495 & 1.4868 & 1.2822 & 1.2687 \\
\hline \multirow{6}{*}{ (d) } & \multirow{3}{*}{1} & 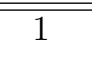 & $\bar{~} 1.0000$ & $\bar{~} 1.0000$ & 1.0000 & $\bar{~} 1.0000$ & $\bar{~} 1.0000$ & 1.0000 \\
\hline & & 2 & 0.9508 & 0.9891 & 0.9861 & 1.0487 & 1.1435 & 1.0837 \\
\hline & & 4 & 0.8036 & 0.7566 & 0.7855 & 1.0686 & 1.3116 & 1.3987 \\
\hline & \multirow{3}{*}{2} & 1 & 0.6927 & 0.6938 & 0.6990 & 0.8384 & 1.0125 & 1.0672 \\
\hline & & 2 & 0.7034 & 0.7080 & 0.7039 & 0.8984 & 1.0898 & 1.1219 \\
\hline & & 4 & 0.7464 & 0.6360 & 0.6049 & 1.1042 & 1.4822 & 1.7349 \\
\hline \multirow{6}{*}{ (e) } & \multirow{3}{*}{1} & $\bar{~} 1$ & 1.0000 & 1.0000 & 1.0000 & 1.0000 & 1.0000 & 1.0000 \\
\hline & & 2 & 0.9598 & 0.8858 & 0.9760 & 1.3243 & 0.9844 & 1.1553 \\
\hline & & 4 & 0.9225 & 0.7550 & 0.8714 & 1.5289 & 1.1056 & 1.4613 \\
\hline & \multirow{3}{*}{2} & 1 & 0.7993 & 0.7593 & 0.8510 & 1.2130 & 1.0426 & 1.3446 \\
\hline & & 2 & 0.8270 & 0.7716 & 0.8487 & 1.4044 & 1.0608 & 1.3821 \\
\hline & & 4 & 0.9127 & 0.7722 & 0.8678 & 1.7733 & 1.1332 & 1.5380 \\
\hline
\end{tabular}


Table 3: Monte Carlo Relative MSE, $\operatorname{MSE}\left(\widehat{\lambda}_{A}\right) / \operatorname{MSE}(O L S)$.

\begin{tabular}{|c|c|c|c|c|c|c|c|c|}
\hline & $\phi$ & $\begin{array}{r}\lambda_{0} \\
L \backslash n\end{array}$ & 96 & $\begin{array}{c}0.4 \\
198\end{array}$ & 392 & 96 & $\begin{array}{c}0.8 \\
198\end{array}$ & 392 \\
\hline \multirow{6}{*}{ (b) } & \multirow{3}{*}{1} & 1 & $\begin{array}{l}1.0000 \\
\end{array}$ & 1.0000 & 1.0000 & $\overline{c 1.0000}$ & 1.0000 & 1.0000 \\
\hline & & 2 & 1.0084 & 0.9958 & 0.9798 & 0.9737 & 0.9868 & 0.9884 \\
\hline & & 4 & 0.3080 & 0.1223 & 0.1051 & 0.1395 & 0.0859 & 0.0644 \\
\hline & \multirow{3}{*}{2} & 1 & 1.9022 & 2.3733 & 2.4731 & 2.1503 & 2.3157 & 2.3996 \\
\hline & & 2 & 1.4770 & 1.9917 & 2.0356 & 1.6565 & 1.8489 & 1.9748 \\
\hline & & 4 & 0.2732 & 0.0773 & 0.0702 & 0.0795 & 0.0480 & 0.0358 \\
\hline \multirow{6}{*}{ (c) } & \multirow{3}{*}{1} & 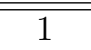 & " & " 1.0000 & " & " & " 1.0000 & 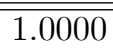 \\
\hline & & 2 & 0.8215 & 0.7971 & 0.8826 & 0.6733 & 0.7395 & 0.8501 \\
\hline & & 4 & 0.5736 & 0.5066 & 0.4587 & 0.5081 & 0.4072 & 0.3929 \\
\hline & \multirow{3}{*}{2} & 1 & 0.6371 & 0.5586 & 0.4922 & 0.5690 & 0.4938 & 0.4491 \\
\hline & & 2 & 0.6581 & 0.5511 & 0.4909 & 0.5653 & 0.4846 & 0.4454 \\
\hline & & 4 & 0.6639 & 0.5666 & 0.4909 & 0.5842 & 0.4877 & 0.4400 \\
\hline \multirow{6}{*}{ (d) } & \multirow{3}{*}{1} & 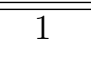 & 1.0000 & 1.0000 & 1.0000 & 1.0000 & " 1.0000 & 1.0000 \\
\hline & & 2 & 0.9482 & 0.9800 & 0.9806 & 0.8952 & 0.9530 & 0.9711 \\
\hline & & 4 & 0.7980 & 0.7372 & 0.7404 & 0.6839 & 0.6907 & 0.6776 \\
\hline & \multirow{3}{*}{2} & 1 & 0.6906 & 0.6787 & 0.6733 & 0.6631 & 0.6488 & 0.6357 \\
\hline & & 2 & 0.6998 & 0.6870 & 0.6734 & 0.6412 & 0.6443 & 0.6318 \\
\hline & & 4 & 0.7405 & 0.5946 & 0.5395 & 0.5899 & 0.5150 & 0.4590 \\
\hline \multirow{6}{*}{ (e) } & \multirow{3}{*}{1} & 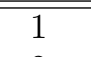 & " 1.0000 & 1.0000 & " 1.0000 & " 1.0000 & " 1.0000 & $\bar{~} 1.0000$ \\
\hline & & 2 & 0.9497 & 0.8930 & 0.9674 & 0.8999 & 0.9239 & 0.9471 \\
\hline & & 4 & 0.9084 & 0.7553 & 0.8017 & 0.8251 & 0.7681 & 0.7261 \\
\hline & \multirow{3}{*}{2} & 1 & 0.7910 & 0.7555 & 0.7848 & 0.7713 & 0.7502 & 0.7123 \\
\hline & & 2 & 0.8156 & 0.7645 & 0.7834 & 0.7780 & 0.7486 & 0.7117 \\
\hline & & 4 & 0.9025 & 0.7690 & 0.7942 & 0.8355 & 0.7675 & 0.7154 \\
\hline
\end{tabular}


Table 4: Monte Carlo Relative Variance, $\operatorname{Var}\left(\widehat{\beta}_{A}\right) / \operatorname{Var}(O L S)$.

\begin{tabular}{|c|c|c|c|c|c|c|c|c|}
\hline & $\phi$ & $\begin{array}{r}\lambda_{0} \\
L \backslash n\end{array}$ & 96 & $\begin{array}{c}0.4 \\
198\end{array}$ & 392 & 96 & $\begin{array}{c}0.8 \\
198\end{array}$ & 392 \\
\hline \multirow{6}{*}{ (b) } & \multirow{3}{*}{1} & 1 & 1.0000 & 1.0000 & 1.0000 & $\overline{c 1.0000}$ & 1.0000 & 1.0000 \\
\hline & & 2 & 1.0009 & 0.9986 & 0.9961 & 1.0058 & 1.0077 & 0.9967 \\
\hline & & 4 & 0.1822 & 0.1671 & 0.1453 & 0.2187 & 0.1965 & 0.1641 \\
\hline & \multirow{3}{*}{2} & 1 & 2.2720 & 2.4539 & 2.5556 & 2.1362 & 2.3412 & 2.4658 \\
\hline & & 2 & 1.7792 & 2.0118 & 2.2734 & 1.7425 & 2.0080 & 2.2346 \\
\hline & & 4 & 0.1163 & 0.1150 & 0.1109 & 0.1423 & 0.1303 & 0.1164 \\
\hline \multirow{6}{*}{ (c) } & \multirow{3}{*}{1} & 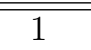 & " & " 1.0000 & " & " & " & 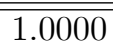 \\
\hline & & 2 & 0.7637 & 0.8297 & 0.9379 & 0.7717 & 0.8387 & 0.9377 \\
\hline & & 4 & 0.5952 & 0.5535 & 0.5717 & 0.6033 & 0.5675 & 0.5765 \\
\hline & \multirow{3}{*}{2} & 1 & 0.6069 & 0.5739 & 0.5537 & 0.6233 & 0.5860 & 0.5596 \\
\hline & & 2 & 0.6211 & 0.5777 & 0.5608 & 0.6339 & 0.5906 & 0.5659 \\
\hline & & 4 & 0.6413 & 0.5854 & 0.5659 & 0.6495 & 0.6078 & 0.5728 \\
\hline \multirow{6}{*}{ (d) } & \multirow{3}{*}{1} & 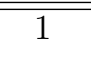 & 1.0000 & 1.0000 & 1.0000 & 1.0000 & " 1.0000 & 1.0000 \\
\hline & & 2 & 0.9771 & 0.9653 & 0.9823 & 0.9843 & 0.9661 & 0.9828 \\
\hline & & 4 & 0.8441 & 0.7839 & 0.7763 & 0.8609 & 0.7968 & 0.7861 \\
\hline & \multirow{3}{*}{2} & 1 & 0.7319 & 0.6929 & 0.6781 & 0.7439 & 0.7110 & 0.6909 \\
\hline & & 2 & 0.7397 & 0.6955 & 0.6781 & 0.7583 & 0.7130 & 0.6914 \\
\hline & & 4 & 0.7470 & 0.6471 & 0.6153 & 0.7864 & 0.6957 & 0.6436 \\
\hline \multirow{6}{*}{ (e) } & \multirow{3}{*}{1} & 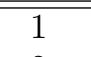 & " 1.0000 & " 1.0000 & " 1.0000 & "1.0000 & " 1.0000 & $\bar{~} 1.0000$ \\
\hline & & 2 & 0.9188 & 0.9485 & 0.9690 & 0.9245 & 0.9508 & 0.9694 \\
\hline & & 4 & 0.8735 & 0.8558 & 0.8035 & 0.8786 & 0.8646 & 0.8098 \\
\hline & \multirow{3}{*}{2} & 1 & 0.8240 & 0.8178 & 0.7820 & 0.8285 & 0.8240 & 0.7930 \\
\hline & & 2 & 0.8385 & 0.8318 & 0.7841 & 0.8507 & 0.8403 & 0.7942 \\
\hline & & 4 & 0.9431 & 0.8807 & 0.7882 & 0.9446 & 0.8862 & 0.8002 \\
\hline
\end{tabular}


Table 5: Monte Carlo Relative $\operatorname{MSE}, \operatorname{MSE}\left(\widehat{\beta}_{A}\right) / \operatorname{MSE}(O L S)$.

\begin{tabular}{|c|c|c|c|c|c|c|c|c|}
\hline & $\phi$ & $\begin{array}{r}\lambda_{0} \\
L \backslash n\end{array}$ & 96 & $\begin{array}{c}0.4 \\
198\end{array}$ & 392 & 96 & $\begin{array}{l}0.8 \\
198\end{array}$ & 392 \\
\hline \multirow{6}{*}{ (b) } & \multirow{3}{*}{1} & 1 & 1.0000 & 1.0000 & 1.0000 & 1.0000 & 1.0000 & 1.0000 \\
\hline & & 2 & 1.0011 & 0.9992 & 0.9960 & 1.0059 & 1.0096 & 0.9962 \\
\hline & & 4 & 0.1823 & 0.1670 & 0.1444 & 0.2188 & 0.1934 & 0.1590 \\
\hline & \multirow{3}{*}{2} & 1 & 2.2720 & 2.4527 & 2.5587 & 2.1362 & 2.3333 & 2.4710 \\
\hline & & 2 & 1.7804 & 2.0153 & 2.2745 & 1.7434 & 2.0073 & 2.2307 \\
\hline & & 4 & 0.1163 & 0.1150 & 0.1102 & 0.1423 & 0.1282 & 0.1127 \\
\hline \multirow{6}{*}{ (c) } & \multirow{3}{*}{1} & 1 & 1.0000 & 1.0000 & 1.0000 & 1.0000 & $\overline{c 1.0000}$ & 1.0000 \\
\hline & & 2 & 0.7637 & 0.8295 & 0.9379 & 0.7717 & 0.8274 & 0.9364 \\
\hline & & 4 & 0.5952 & 0.5532 & 0.5719 & 0.6033 & 0.5603 & 0.5737 \\
\hline & \multirow{3}{*}{2} & 1 & 0.6068 & 0.5736 & 0.5539 & 0.6232 & 0.5804 & 0.5575 \\
\hline & & 2 & 0.6210 & 0.5773 & 0.5610 & 0.6339 & 0.5841 & 0.5637 \\
\hline & & 4 & 0.6420 & 0.5852 & 0.5661 & 0.6503 & 0.6001 & 0.5704 \\
\hline \multirow{6}{*}{ (d) } & \multirow{3}{*}{1} & 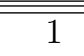 & 1.0000 & 1.0000 & 1.0000 & "1.0000 & "1.0000 & 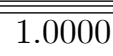 \\
\hline & & 2 & 0.9773 & 0.9640 & 0.9826 & 0.9849 & 0.9609 & 0.9829 \\
\hline & & 4 & 0.8446 & 0.7830 & 0.7765 & 0.8618 & 0.7904 & 0.7839 \\
\hline & \multirow{3}{*}{2} & 1 & 0.7322 & 0.6928 & 0.6788 & 0.7446 & 0.7092 & 0.6909 \\
\hline & & 2 & 0.7400 & 0.6947 & 0.6794 & 0.7590 & 0.7087 & 0.6924 \\
\hline & & 4 & 0.7477 & 0.6461 & 0.6171 & 0.7875 & 0.6867 & 0.6426 \\
\hline \multirow{6}{*}{ (e) } & \multirow{3}{*}{1} & 1 & 1.0000 & 1.0000 & 1.0000 & 1.0000 & 1.0000 & 1.0000 \\
\hline & & 2 & 0.9187 & 0.9485 & 0.9694 & 0.9244 & 0.9504 & 0.9703 \\
\hline & & 4 & 0.8733 & 0.8557 & 0.8045 & 0.8785 & 0.8630 & 0.8112 \\
\hline & \multirow{3}{*}{2} & 1 & 0.8239 & 0.8178 & 0.7830 & 0.8284 & 0.8226 & 0.7944 \\
\hline & & 2 & 0.8383 & 0.8318 & 0.7853 & 0.8506 & 0.8384 & 0.7957 \\
\hline & & 4 & 0.9428 & 0.8811 & 0.7891 & 0.9441 & 0.8825 & 0.8000 \\
\hline
\end{tabular}


Table 6: Monte Carlo Relative Variance and MSE of $\widehat{\lambda}_{B}, \lambda_{0}=0.4$.

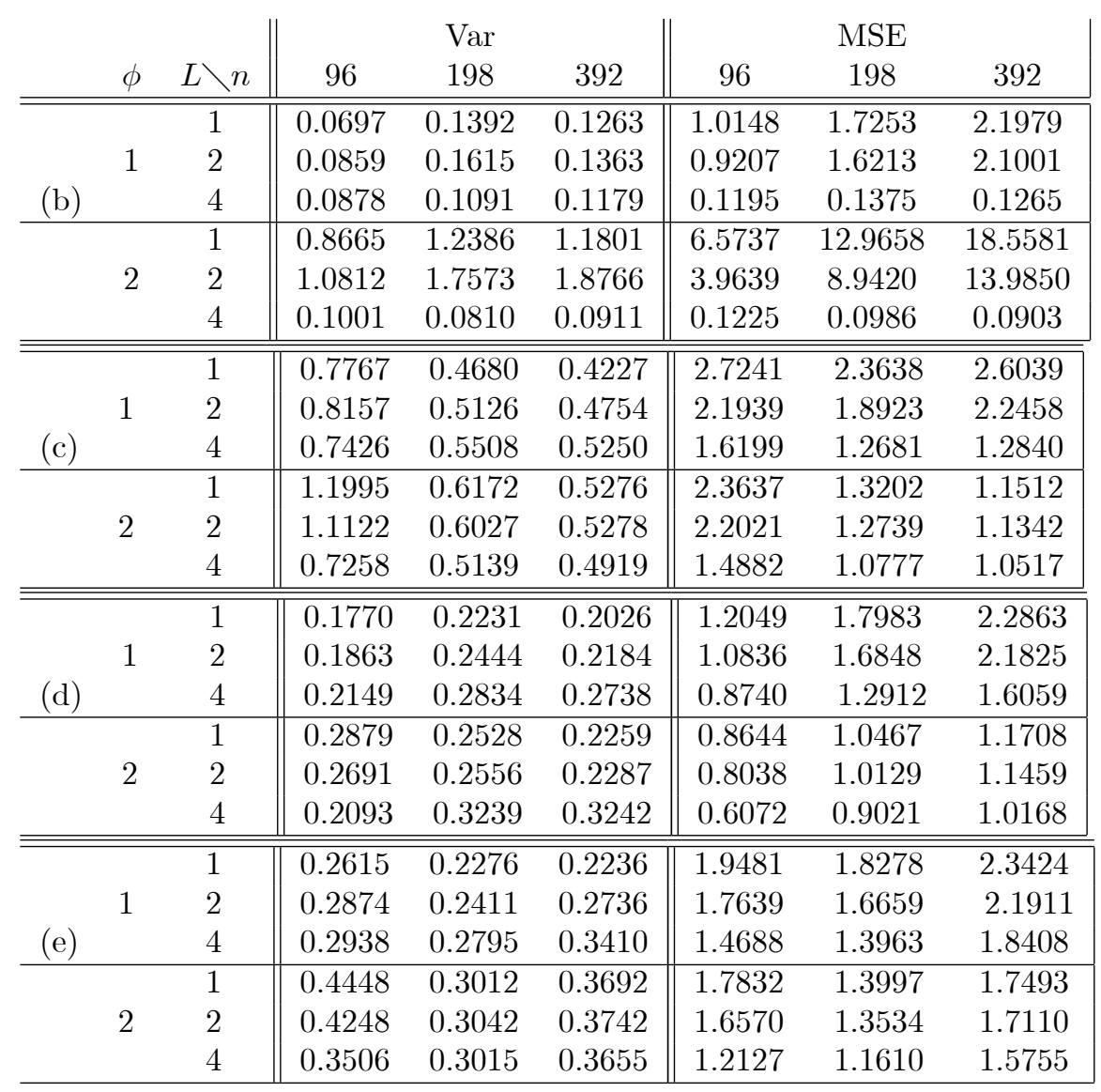


Table 7: Monte Carlo Relative Variance and MSE of $\widehat{\beta}_{B}, \lambda_{0}=0.4$.

\begin{tabular}{|c|c|c|c|c|c|c|c|c|}
\hline & & & & Var & & & MSE & \\
\hline & $\phi$ & $L \backslash n$ & 96 & 198 & 392 & 96 & 198 & 392 \\
\hline & & 1 & 0.9205 & 1.0670 & 1.0146 & 0.9322 & 1.0723 & 1.0082 \\
\hline & 1 & 2 & 0.9244 & 1.0629 & 1.0105 & 0.9368 & 1.0664 & 1.0041 \\
\hline (b) & & 4 & 0.1752 & 0.1693 & 0.1447 & 0.1761 & 0.1689 & 0.1443 \\
\hline & & 1 & 2.0524 & 2.8491 & 2.7023 & 2.1118 & 2.9154 & 2.6935 \\
\hline & 2 & 2 & 1.6370 & 2.3079 & 2.3982 & 1.6769 & 2.3323 & 2.3881 \\
\hline & & 4 & 0.1141 & 0.1161 & 0.1106 & 0.1145 & 0.1158 & 0.1100 \\
\hline & & 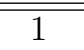 & 0.9455 & " 1.0254 & " 1.0339 & 0.9653 & 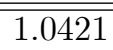 & 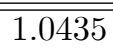 \\
\hline & 1 & 2 & 0.7365 & 0.8505 & 0.9685 & 0.7498 & 0.8725 & 0.9771 \\
\hline (c) & & 4 & 0.5785 & 0.5561 & 0.5835 & 0.5873 & 0.5653 & 0.5888 \\
\hline & & 1 & 0.5926 & 0.5783 & 0.5687 & 0.6028 & 0.5884 & 0.5737 \\
\hline & 2 & 2 & 0.6057 & 0.5824 & 0.5760 & 0.6153 & 0.5925 & 0.5810 \\
\hline & & 4 & 0.6272 & 0.5861 & 0.5796 & 0.6386 & 0.5960 & 0.5845 \\
\hline & & 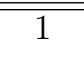 & $\bar{~} 0.9210$ & 1.0588 & " 1.0240 & 0.9297 & 1.0609 & 1.0168 \\
\hline & 1 & 2 & 0.9092 & 1.0219 & 1.0043 & 0.9151 & 1.0255 & 0.9972 \\
\hline (d) & & 4 & 0.7942 & 0.8188 & 0.7880 & 0.7973 & 0.8203 & 0.7824 \\
\hline & & 1 & 0.6809 & 0.7219 & 0.6865 & 0.6842 & 0.7219 & 0.6817 \\
\hline & 2 & 2 & 0.6929 & 0.7224 & 0.6876 & 0.6956 & 0.7231 & 0.6829 \\
\hline & & 4 & 0.7114 & 0.6687 & 0.6191 & 0.7124 & 0.6688 & 0.6152 \\
\hline & & $\bar{~} 1$ & 0.9216 & 1.0587 & 1.0305 & 0.9300 & 1.0817 & 1.0305 \\
\hline & 1 & 2 & 0.8496 & 0.9941 & 0.9974 & 0.8572 & 1.0156 & 0.9969 \\
\hline (e) & & 4 & 0.8151 & 0.8931 & 0.8237 & 0.8221 & 0.9098 & 0.8228 \\
\hline & & 1 & 0.7657 & 0.8614 & 0.7983 & 0.7726 & 0.8782 & 0.7974 \\
\hline & 2 & 2 & 0.7822 & 0.8730 & 0.8019 & 0.7894 & 0.8890 & 0.8009 \\
\hline & & 4 & 0.8860 & 0.9198 & 0.8068 & 0.8922 & 0.9371 & 0.8058 \\
\hline
\end{tabular}




\section{References}

Arbia, G., 2006 Spatial econometrics: statistical foundations and applications to regional analysis. (Springer-Verlag, Berlin).

Beran, R., 1976 Adaptive estimates for autoregressive processes. Annals of the Institute of Statistical Mathematics 26, 77-89.

Bickel, P., 1982 On adaptive estimation. Annals of Statistics 10, 647-671.

Case, A.C., 1991 Neighbourhood influence and technological change. Regional Science and Urban Economics 22, 491-508.

Freud, G., 1971 Orthogonal polynomials. (Pergamon Press, Oxford).

Kelejian, H.H., Prucha, I.R., 1998 A generalized spatial two-stage least squares procedure for estimating a spatial autoregressive model with autoregressive disturbances. Journal of Real Estate Finance and Economics 17, 99-121.

Kelejian, H.H., Prucha, I.R., 1999 A generalized moments estimator for the autoregressive parameter in a spatial model. International Economic Review 40, 509-533.

Kelejian, H.H., Prucha, I.R., Yuzefovich, Y., 2003 Instrumental variable estimation of a spatial autoregressive model with autoregressive disturbances: large and small sample results. Preprint.

Lee, L.F., 2002 Consistency and efficiency of least squares estimation for mixed regressive, spatial autoregressive models. Econometric Theory 18, 252277.

Lee, L.F., 2003 Best spatial two-stage least squares estimators for a spatial autoregressive model with autoregressive disturbances. Econometric Reviews $22,307-335$.

Lee, L.F., 2004 Asymptotic distributions of quasi-maximum likelihood estimates for spatial autoregressive models. Econometrica 72, 1899-1925.

Newey, W.K., 1988 Adaptive estimation of regression models via moment restrictions. Journal of Econometrics 38, 301-339.

Pőtscher, B.M., Prucha, I.R., 1986 A class of partially adaptive one-step M-estimates for the non-linear regression model with dependent observations. Journal of Econometrics 32, 219-251.

Robinson, P.M., 1988 The stochastic difference between econometric statistics. Econometrica 56, 531-548.

Robinson, P.M., 2005 Efficiency improvements in inference on stationary and nonstationary fractional time series. Annals of Statistics 33, 1800-1842.

Stone, C.J., 1975 Adaptive maximum likelihood estimators of a location parameter. Annals of Statistics 2, 267-289.

Whittle, P., 1954 On stationary processes in the plane. Biometrika 41, 434449 . 